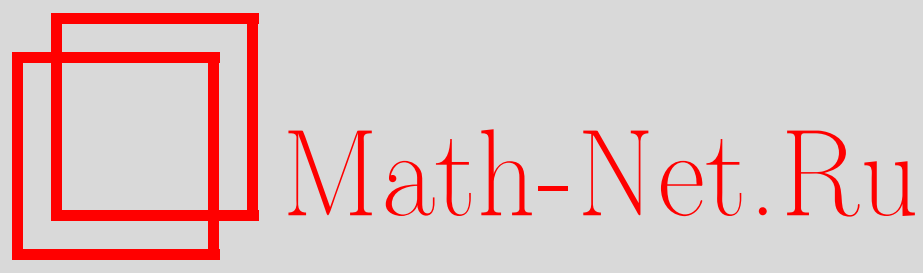

Р. Луцци, А. Р. Васкончеллос, Х. Г. Рамос, К. Г. Родригез, Статистическая термодинамика необратимых процессов в рамках метода неравновесного статистического оператора Зубарева, ТМФ, 2018, том 194, номер 1, 7-38

DOI: https://doi.org/10.4213/tmf9362

Использование Общероссийского математического портала Math-Net.Ru подразумевает, что вы прочитали и согласны с пользовательским соглашением http://www . mathnet.ru/rus/agreement

Параметры загрузки:

IP : 54.237 .59 .107

26 апреля 2023 г., $11: 46: 12$

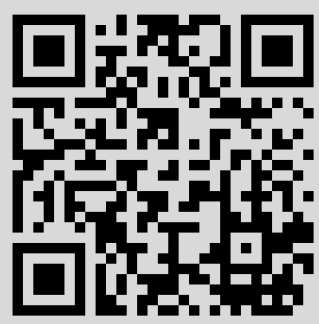




\title{
ФИЗИКА
}

Том 194, № 1

январь, 2018

(C) 2018 г.

Р. Луцци*, А.Р. Васкончеллос *, Х. Г. Рамос*, К. Г. Родригез ${ }^{\dagger}$

\section{СТАТИСТИЧЕСКАЯ ТЕРМОДИНАМИКА НЕОБРАТИМЫХ ПРОЦЕССОВ В РАМКАХ МЕТОДА НЕРАВНОВЕСНОГО СТАТИСТИЧЕСКОГО ОПЕРАТОРА ЗУБАРЕВА}

\begin{abstract}
Описывается формализм статистической термодинамики необратимых процессов, построенный на основе метода неравновесного статистического оператора Зубарева - мощного и универсального способа исследования самых разнообразных физических явлений. Представлен краткий обзор формализма статистических ансамблей и статистической термодинамики необратимых процессов. Первый из них может быть построен либо на основе эвристического подхода, либо в рамках теории информации, следуя схеме научных предсказаний Джеффри-Джейнса. Оба подхода были использованы Д. Н. Зубаревым и его школой при формулировке метода неравновесного статистического оператора. Описываются главные черты статистической термодинамики необратимых процессов и обсуждаются некоторые особые соображения, принадлежащие нескольким авторам. Кратко описан способ вывода термодинамического принципа неопределенности, предложенный Розенфельдом, Бором и Пригожиным.
\end{abstract}

Ключевые слова: термодинамика необратимых процессов, теория информации, статистическая механика.

DOI: https://doi.org/10.4213/tmf9362

\section{1. ВВЕДЕНИЕ}

Термодинамику, так же как и статистическую механику, можно разделить на две части, одна из которых соответствует изучению систем в равновесии, а другая неравновесным ситуациям. Последний случай относится к термодинамике необратимых процессов, причем можно выделить два различных и важных частных случая:

Авторы благодарят за частичную финансовую поддержку, полученную от São Paulo State Research Agency (FAPESP) и Goiás State Research Agency (FAPEG).

*Department of Condensed Matter Physics, Institute of Physics "Gleb Wataghin", State University of Campinas (UNICAMP), Campinas, Brazil

${ }^{\dagger}$ Escola de Ciências Exatas e da Computação, Pontifícia Universidade Católica de Goiás, Goiânia, Goiás, Brazil. E-mail: cloves@pucgoias.edu.br 
так называемый линейный режим и нелинейный режим. Очевидно, что термодинамика нелинейных необратимых процессов является разделом модной в настоящее время нелинейной физики. Более точно, ее можно рассматривать как частный случай развивающейся теории сложных динамических систем [1]-[4], в ряде аспектов имеющей отношение к самоорганизации в открытых диссипативных системах вдали от равновесия и, возможно, к возникновению, функционированию и развитию жизни. Пригожин [5] внес значительный вклад в эту новую концепцию сложности и был одним из ее основателей.

Здесь мы опишем то, что можно назвать статистической термодинамикой необратимых процессов (СТНП) [6]-[12], т. е. неравновесную термодинамику, основанную на формализме неравновесных статистических ансамблей (ФНСА) [13]-[26].

В разделе 2 кратко описывается ФНСА. Раздел 3 содержит обзор СТНП, а затем в разделе 4 рассматривается несколько общих аспектов СТНП: некоторые свойства статистической энтропии и ее производства, неравновесные уравнения состояния, обобщенная $\mathcal{H}$-теорема, критерии эволюции и (не)устойчивости, обобщенные соотношения Клаузиуса, флуктуации, соотношения типа соотношений Максвелла и соотношения Больцмана для информационной статистической энтропии. Вывод в рамках СТНП термодинамического принципа неопределенности в форме, предложенной Розенфельдом, Бором и Пригожиным, кратко рассматривается в разделе 5. В разделах 6 и 7 приводятся заключительные соображения.

\section{2. ТЕРМОДИНАМИКА НЕОБРАТИМЫХ ПРОЦЕССОВ И МЕТОД НЕРАВНОВЕСНОГО СТАТИСТИЧЕСКОГО OПЕРАТОРА ЗУБАРЕВА}

Напомним, что неравновесная термодинамика представляет собой макроскопическую и феноменологическую теорию, которая занимается состояниями и процессами в слабо или сильно неравновесных системах, причем равновесие рассматривается как состояние, в котором параметры состояния не изменяются со временем и отсутствуют потоки динамических величин на границах системы. Неравновесная термодинамика изучает, главным образом, процессы переноса и релаксационные явления в непрерывной среде, а также возникновение стационарных состояний, их устойчивость и сопровождающие релаксационные явления.

Обратимся теперь к вопросу о микроскопическом подходе к термодинамике необратимых процессов в $\Phi$ НСА, т. е. в формализме, основанном на методе неравновесного статистического оператора (метод НСО) Зубарева, начав с краткого описания последнего.

ФНСА является очень мощным, компактным, основанным на разумных принципах и изящным формализмом для изучения систем, сколь угодно далеких от равновесия. Цванциг [27] утверждал, что этот формализм “имеет безусловно наиболее привлекательную структуру и может оказаться наиболее эффективным методом изучения нелинейных процессов переноса".

Дальнейшие события подтвердили предсказание Цванцига. Современная структура формализма представляет собой далеко идущее расширение и обобщение более ранних важных подходов, среди которых можно отметить работы Кирквуда [28], Грина [29], Мори, Оппенгейма, Росса [30], Мори [31] и Цванцига [32]. К ФНСА 
подходили с разных точек зрения: некоторые основаны на эвристических соображениях, другие - на технике операторов проектирования (первый подход восходит к Кирквуду и Грину, а второй - к Цванцигу и Мори).

Прежде всего нужно отметить, что всякий раз при рассмотрении неравновесных систем следует тщательно учитывать несколько важных обстоятельств.

1. Выбор базисных переменных (совершенно отличный от случая равновесия, когда достаточно взять набор интегралов движения), который должен быть основан на анализе того, какого типа макроскопические измерения и процессы реально возможны. Более того, нужно обратить внимание не только на то, что можно наблюдать, но также на характер и ожидаемый вид уравнений эволюции для этих переменных (см., например, [27]-[32]). Заметим также, что даже если на начальной стадии нам потребовалось бы ввести все наблюдаемые для системы и их случайные отклонения, то с течением времени становится возможным все более сокращенное описание эволюции, когда вступают в силу принцип ослабления корреляций Боголюбова и соответствующая иерархия времен релаксации [33].

2. Вопрос о необратимости (или стрела времени Эддингтона), по поводу которого Пайерлс [34] утверждал следующее: “В каждом теоретическом исследовании процессов переноса нужно ясно понимать, в каком месте была введена необратимость. Если она не введена, теория неверна. Подход, в котором сохранена симметрия относительно обращения времени, неизбежно дает нулевое или бесконечное значение для любого коэффициента переноса. Если мы не видим ясно, где была введена необратимость, то мы не понимаем, что мы делаем".

3. Следует ввести гистерезис, т. е. идею о том, что нужно учитывать предшествующую динамику системы (или эффекты памяти) на всем временно́м интервале от начального макросостояния в данном эксперименте, скажем в момент $t_{0}$, до момента $t$, когда производится измерение. Это чрезвычайно важное обстоятельство в случае диссипативных систем, как подчеркивалось, среди прочих, Кирквудом, Грином, Мори и Цванцигом [28]-[32].

Что касается п. 1, т. е. вопроса о выборе базисных переменных, которые, как уже отмечалось, существенно отличаются от переменных в случае равновесия, то сразу после того, как открытая система из $N$ частиц, находящаяся в контакте с внешними источниками и резервуарами, была выведена из равновесия, ее состояние пришлось бы характеризовать всеми наблюдаемыми, вводя в конечном итоге прямые и перекрестные корреляции. Это означает, однако, что следует обратиться к одночастичному $\left(\hat{n}_{1}\right)$ и двухчастичному $\left(\hat{n}_{2}\right)$ динамическим операторам, поскольку на микроскопическом уровне все наблюдаемые величины и их корреляции можно выразить через эти динамические операторы. Описание механических состояний в терминах приведенных операторов плотности можно найти в уже ставшей классической статье Фано [35].

В квантовой механике одно- и двухчастичные операторы плотности имеют вид

$$
\begin{aligned}
\hat{n}_{1}\left(\mathbf{r}, \sigma, \mathbf{r}^{\prime}, \sigma^{\prime}\right) & =\psi_{\sigma}^{\dagger}(\mathbf{r}) \psi_{\sigma^{\prime}}\left(\mathbf{r}^{\prime}\right), \\
\hat{n}_{2}\left(\mathbf{r}_{1}, \sigma_{1}, \mathbf{r}_{2}, \sigma_{2}, \mathbf{r}_{2}^{\prime}, \sigma_{2}^{\prime}, \mathbf{r}_{1}^{\prime}, \sigma_{1}^{\prime}\right) & =\psi_{\sigma_{1}}^{\dagger}\left(\mathbf{r}_{1}\right) \psi_{\sigma_{2}}^{\dagger}\left(\mathbf{r}_{2}\right) \psi_{\sigma_{2}^{\prime}}\left(\mathbf{r}_{2}^{\prime}\right) \psi_{\sigma_{1}^{\prime}}\left(\mathbf{r}_{1}^{\prime}\right),
\end{aligned}
$$

где $\sigma$ - спиновый индекс, $\psi$ и $\psi^{\dagger}$ - одночастичные полевые операторы в представлении вторичного квантования (их превосходное дидактическое описание имеется в статье Робертсона [36]). 
Эти операторы можно выразить через операторы уничтожения $c_{\mathbf{k} \sigma}$ и операторы рождения $c_{\mathbf{k} \sigma}^{\dagger}$ в одночастичных состояниях, скажем с волновыми функциями $\varphi_{\mathbf{k} \sigma}(\mathbf{r})$ и уровнями энергии $\epsilon_{\mathbf{k} \sigma}$ :

$$
\psi_{\sigma}(\mathbf{r})=\sum_{\mathbf{k}} \varphi_{\mathbf{k} \sigma}(\mathbf{r}) c_{\mathbf{k} \sigma}, \quad \psi_{\sigma}^{\dagger}(\mathbf{r})=\sum_{\mathbf{k}} \varphi_{\mathbf{k} \sigma}^{*}(\mathbf{r}) c_{\mathbf{k} \sigma}^{\dagger}
$$

Это представление очень удобно, так как оно вводит описание в обратном $\mathbf{k}$-пространстве, которое обладает практическими преимуществами для вычислений и физической интерпретации. Действуя таким образом, мы можем ввести вместо $\hat{n}_{1}$ и $\hat{n}_{2}$ из (1) матрицы, составленные из величин

$$
\left\{\left\{c_{\mathbf{k} \sigma}^{\dagger} c_{\mathbf{k}^{\prime} \sigma^{\prime}}\right\},\left\{c_{\mathbf{k} \sigma}^{\dagger} c_{\mathbf{k}^{\prime} \sigma^{\prime}}^{\dagger} c_{\mathbf{k}_{1} \sigma_{1}} c_{\mathbf{k}_{2} \sigma_{2}}\right\}\right\}
$$

Следовательно, любая одночастичная механическая наблюдаемая может быть выражена через величины

$$
\left\{\hat{n}_{\mathbf{k} \sigma \sigma^{\prime}}=c_{\mathbf{k} \sigma}^{\dagger} c_{\mathbf{k} \sigma^{\prime}}, \hat{n}_{\mathbf{k} \mathbf{Q} \sigma \sigma^{\prime}}=c_{\mathbf{k}+\frac{1}{2} \mathbf{Q}, \sigma}^{\dagger} c_{\mathbf{k}-\frac{1}{2} \mathbf{Q}, \sigma^{\prime}}\right\}
$$

c $\mathbf{Q} \neq 0$, где мы разделили диагональные и недиагональные члены: первые представляют собой так называемые заселенности, а вторые - когерентности [37] (в то время как заселенности используются при описании глобальных свойств (или однородных состояний), когерентности описывают локальные свойства (или неоднородные состояния)). Любая одночастичная механическая наблюдаемая может быть записана в представлении вторичного квантования в хорошо известном виде [38], [39]:

$$
\hat{A}=\sum_{\mathbf{k} \sigma, \mathbf{k}^{\prime} \sigma^{\prime}}\left\langle\mathbf{k} \sigma|\hat{A}| \mathbf{k}^{\prime} \sigma^{\prime}\right\rangle c_{\mathbf{k} \sigma}^{\dagger} c_{\mathbf{k}^{\prime} \sigma^{\prime}},
$$

куда входят матричные элементы $\hat{A}$ между одночастичными состояниями $\langle\mathbf{k} \sigma|$ и $\left|\mathbf{k}^{\prime} \sigma^{\prime}\right\rangle$.

Для двухчастичных механических наблюдаемых, используя в (3) замену индексов $\mathbf{k}$ на $\mathbf{k}+\frac{1}{2} \mathbf{Q}, \mathbf{k}^{\prime}$ на $\mathbf{k}^{\prime}-\frac{1}{2} \mathbf{Q}^{\prime}, \mathbf{k}_{1}$ на $\mathbf{k}-\frac{1}{2} \mathbf{Q}, \mathbf{k}_{2}$ на $\mathbf{k}^{\prime}+\frac{1}{2} \mathbf{Q}^{\prime}$, имеем

$$
\hat{n}_{\mathbf{k} \sigma_{1}, \mathbf{k}^{\prime} \sigma_{1}^{\prime}, \mathbf{k}^{\prime} \sigma_{2}, \mathbf{k} \sigma_{2}^{\prime}}=c_{\mathbf{k} \sigma_{1}}^{\dagger} c_{\mathbf{k}^{\prime} \sigma_{1}^{\prime}}^{\dagger} c_{\mathbf{k}^{\prime} \sigma_{2}} c_{\mathbf{k} \sigma_{2}^{\prime}}
$$

для $\mathbf{Q}, \mathbf{Q}^{\prime}=0$ и

$$
\hat{n}_{\mathbf{k}+\frac{1}{2} \mathbf{Q} \sigma_{1}, \mathbf{k}^{\prime}-\frac{1}{2} \mathbf{Q}^{\prime} \sigma_{1}^{\prime}, \mathbf{k}-\frac{1}{2} \mathbf{Q} \sigma_{2}, \mathbf{k}^{\prime}+\frac{1}{2} \mathbf{Q}^{\prime} \sigma_{2}^{\prime}}=c_{\mathbf{k}+\frac{1}{2} \mathbf{Q} \sigma_{1}}^{\dagger} c_{\mathbf{k}^{\prime}-\frac{1}{2} \mathbf{Q}^{\prime} \sigma_{1}^{\prime}}^{\dagger} c_{\mathbf{k}-\frac{1}{2} \mathbf{Q} \sigma_{2}} c_{\mathbf{k}^{\prime}+\frac{1}{2} \mathbf{Q}^{\prime} \sigma_{2}^{\prime}}
$$

для $\mathbf{Q}, \mathbf{Q}^{\prime} \neq 0$. Любая двухчастичная наблюдаемая принимает вид [38], [39]

$$
\begin{aligned}
\hat{B}= & \sum_{\substack{\mathbf{k}, \mathbf{k}^{\prime}, \mathbf{Q}, \mathbf{Q}^{\prime}, \sigma_{1}, \sigma_{1}^{\prime}, \sigma_{2}, \sigma_{2}^{\prime}}}\left\langle\mathbf{k}+\frac{1}{2} \mathbf{Q} \sigma_{1}, \mathbf{k}^{\prime}-\frac{1}{2} \mathbf{Q}^{\prime} \sigma_{2}|\hat{B}| \mathbf{k}^{\prime}+\frac{1}{2} \mathbf{Q}^{\prime} \sigma_{2}^{\prime}, \mathbf{k}-\frac{1}{2} \mathbf{Q} \sigma_{1}^{\prime}\right\rangle \times \\
& \times\left[\hat{n}_{\mathbf{k} \mathbf{Q} \sigma_{1} \sigma_{1}^{\prime}} \hat{n}_{\mathbf{k}^{\prime} \mathbf{Q}^{\prime} \sigma_{2} \sigma_{2}^{\prime}}^{\dagger} \mp \delta_{\mathbf{k} \mathbf{Q} \sigma_{1} \sigma_{1}^{\prime}, \mathbf{k}^{\prime} \mathbf{Q}^{\prime} \sigma_{2} \sigma_{2}^{\prime}} c_{\mathbf{k}+\frac{1}{2} \mathbf{Q} \sigma}^{\dagger} c_{\mathbf{k}^{\prime}+\frac{1}{2} \mathbf{Q}^{\prime} \sigma_{1}^{\prime}}\right],
\end{aligned}
$$

где мы заменили порядок в произведении четырех операторов в (7) так, чтобы один член имел вид произведения двух операторов типа $\hat{n}_{1}$, а другой включал $\hat{n}_{1}$, уже 
введенный в (4); знак минус относится к случаю фермионов, а знак полюс - к бозонам. Например, кулоновское взаимодействие между парами электронов дается формулой

$$
\widehat{H}_{C}=\sum_{\mathbf{Q} \neq 0} \mathcal{V}(\mathbf{Q})\left[\hat{n}_{\mathbf{Q}} \hat{n}_{\mathbf{Q}}^{\dagger}-\widehat{N}\right],
$$

где, если в качестве полного набора одночастичных квантовых состояний выбраны плоские волны, $\mathcal{V}(\mathbf{Q})=4 \pi e^{2} / V Q^{2}(V$ - объем системы $)$,

$$
\hat{n}_{\mathbf{Q}}=\sum_{\mathbf{k}, \sigma_{1}, \sigma_{1}^{\prime}} \delta_{\sigma_{1} \sigma_{1}^{\prime}} \hat{n}_{\mathbf{k} \mathbf{Q} \sigma_{1} \sigma_{1}^{\prime}}
$$

- фурье-образ (по волновому вектору Q) оператора плотности заряда (в единицах заряда электрона), а $\widehat{N}=\sum_{\mathbf{k} \sigma} c_{\mathbf{k} \sigma}^{\dagger} c_{\mathbf{k} \sigma}-$ оператор числа частиц.

Обратим внимание на то, что в случае, когда частицы являются бозонами, могут существовать когерентные состояния и тогда для описания системы бозонов (например, когерентных фотонов в лазере, когерентных фононов в полупроводниках и т. д.) к одно- и двухчастичным операторам плотности нужно добавить индивидуальные амплитуды $c_{\mathbf{k} \sigma}$ и $c_{\mathbf{k} \sigma}^{\dagger}$. Когерентные состояния являются собственными состояниями операторов уничтожения и рождения [40]. Кроме того, бывает также необходимо рассматривать пары Гугенхольца-Горькова, т. е. $c_{\mathbf{k}} c_{\mathbf{k}^{\prime}}$ и $c_{\mathbf{k}}^{\dagger} c_{\mathbf{k}^{\prime}}^{\dagger}$ [41].

Возвращаясь к вопросу о выборе базисных переменных и опираясь на приведенное выше обсуждение, отметим, что для описания макросостояния системы в терминах всех механических наблюдаемых возможна альтернативная формулировка, в которой используются одно- и двухчастичные матрицы плотности. Следовательно, с одной стороны, неравновесный статистический оператор, скажем $\mathcal{R}_{\varepsilon}(t)$, зависит от этих величин, а с другой стороны, макропеременными, описывающими неравновесное термодинамическое состояние системы, являются средние значения тех же самых величин по неравновесному ансамблю, а именно

$$
n_{\mathbf{k}}(t)=\operatorname{Tr}\left\{\hat{n}_{\mathbf{k}} \mathcal{R}_{\varepsilon}(t)\right\}, \quad n_{\mathbf{k} \mathbf{Q}}(t)=\operatorname{Tr}\left\{\hat{n}_{\mathbf{k} \mathbf{Q}} \mathcal{R}_{\varepsilon}(t)\right\}
$$

с $\mathbf{Q} \neq 0$ и

$$
n_{\mathbf{k Q}, \mathbf{k}^{\prime} \mathbf{Q}^{\prime}}(t)=\operatorname{Tr}\left\{\hat{n}_{\mathbf{k} \mathbf{Q}} \hat{n}_{\mathbf{k}^{\prime} \mathbf{Q}^{\prime}}^{\dagger} \mathcal{R}_{\varepsilon}(t)\right\}
$$

без ограничений на $\mathbf{Q}$ и $\mathbf{Q}^{\prime}$. Для простоты мы опустили спиновые индексы. Заметим также, что выбор $\hat{n}_{2}$ в виде (12) вместо общего выражения (7) следует из замечания после формулы (8) о том, что можно изменить порядок в произведении четырех операторов, используя коммутационные соотношения для фермионов и бозонов. Разность между двумя выражениями содержит только одночастичный динамический оператор, уже введенный в (4).

Что касается п. 2, то Крылов [42] отмечал, что всегда существует физическое взаимодействие между системой, над которой производится измерение, и внешним миром; оно постоянно "выбивает" систему из ее точного микросостояния. Таким образом, нестабильность траекторий и неустранимое взаимодействие с окружением гарантирует применение "грубо приготовленного" макроскопического описания. В отсутствие подходящего способа учесть подобные эффекты приходится обратиться к тому, что в логике называется интервенционистским подходом, который опирается на вмешательство такого неустранимого процесса хаотизации, проводящего к асимметричной эволюции макросостояния. 
"Вмешательство" заключается в том, что в уравнение Лиувилля для статистического оператора изолированной системы вводится специальный источник, учитывающий “эффект перемешивания” Крылова, в следующей форме (для логарифма статистического оператора):

$$
\frac{\partial}{\partial t} \ln \mathcal{R}_{\varepsilon}(t)+\frac{1}{i \hbar}\left[\ln \mathcal{R}_{\varepsilon}(t), \widehat{H}\right]=-\varepsilon\left[\ln \mathcal{R}_{\varepsilon}(t)-\ln \overline{\mathcal{R}}(t, 0)\right]
$$

где параметр $\varepsilon$ (играющий роль обратного времени релаксации) стремится к +0 после того, как произведено вычисление средних значений. Такой неоднородный член, добавленный в уравнение Лиувилля, выражает постоянную тенденцию релаксации статистических операторов к особому статистическому оператору $\overline{\mathcal{R}}$, который, как показано ниже, соответствует условию мгновенного квазиравновесия.

Видно, что (13) представляет собой обычное уравнение Лиувилля, но с бесконечно малым источником, который обеспечивает нарушение симметрии относительно обращения времени и является ответственным за отбрасывание подгруппы опережающих решений [43]-[45]. Решение уравнения (13) описывается распределением Пуассона, и выражение в момент времени $t$ получается в результате усреднения по всем $t^{\prime}$ в интервале $\left(t_{0}, t\right)$ :

$$
\mathcal{R}_{\varepsilon}(t)=\exp \left\{-\widehat{S}(t, 0)+\int_{t_{0}}^{t} d t^{\prime} e^{\varepsilon\left(t^{\prime}-t\right)} \frac{d}{d t^{\prime}} \widehat{S}\left(t^{\prime}, t^{\prime}-t\right)\right\},
$$

где

$$
\begin{aligned}
\widehat{S}(t, 0) & =-\ln \overline{\mathcal{R}}(t, 0), \\
\widehat{S}\left(t^{\prime}, t^{\prime}-t\right) & =e^{-\left(t^{\prime}-t\right) \widehat{H} / i \hbar} \widehat{S}\left(t^{\prime}, 0\right) e^{\left(t^{\prime}-t\right) \widehat{H} / i \hbar},
\end{aligned}
$$

и начальное условие в момент времени $t_{0}$, начиная с которого применяется формализм, имеет вид

$$
\mathcal{R}_{\varepsilon}\left(t_{0}\right)=\overline{\mathcal{R}}\left(t_{0}, 0\right) .
$$

Обычно момент начала статистического описания $t_{0}$ выбирают в отдаленном прошлом $\left(t_{0} \rightarrow-\infty\right)$, вводя адиабатическое включение процесса релаксации, и в $(14)$ интегрирование по времени в интервале $\left(t_{0}, t\right)$ является взвешенным с ядром $e^{\varepsilon\left(t^{\prime}-t\right)}$. Наличие такого ядра приводит к исчезающей истории, по мере того как макросостояние системы эволюционирует от граничного условия (16) при $t_{0} \rightarrow-\infty$ (результат присутствия в (14) экспоненциального множителя, который учитывает диссипативный характер эволюции состояния системы, - факт, известный из кинетической теории [13]-[24], [46]-[54], что ясно свидетельствует о наличии процесса с затухающей памятъю). Кроме того, во многих случаях можно считать, что полная система включает интересующую нас систему (с которой проводится эксперимент) и идеальные резервуары. Таким образом, можно записать

$$
\mathcal{R}_{\varepsilon}(t)=\varrho_{\varepsilon}(t) \times \varrho_{R},
$$

где $\varrho_{\varepsilon}(t)$ - статистический оператор неравновесной системы, $\varrho_{R}-$ стационарный статистический оператор идеальных резервуаров, причем $\varrho_{\varepsilon}(t)$ дается формулой

$$
\varrho_{\varepsilon}(t)=\exp \left\{-\widehat{S}(t, 0)+\int_{-\infty}^{t} d t^{\prime} e^{\varepsilon\left(t^{\prime}-t\right)} \frac{d}{d t^{\prime}} \widehat{S}\left(t^{\prime}, t^{\prime}-t\right)\right\}
$$


и имеет начальное значение $\bar{\varrho}\left(t_{0}, 0\right)\left(t_{0} \rightarrow-\infty\right)$, где

$$
\widehat{S}(t, 0)=-\ln \bar{\varrho}(t, 0) .
$$

Наконец, нужно найти вспомогательный статистический оператор $\varrho(t, 0)$. Он определяет в момент времени $t$ мгновенное распределение, которое описывает "замороженное" равновесие при заданном макроскопическом состоянии системы, и по этой причине его иногда называют квазиравновесным статистическим оператором. На основе сказанного выше или, альтернативно, следуя процедуре принципа условного экстремума [17], [19], [55]-[58] и рассматривая в качестве примера случай полупроводников, мы вводим только одночастичный динамический оператор для электронов (в одночастичных блоховских состояниях; более точно, в зонах электронов проводимости и дырок) и для фононов отдельных мод различных ветвей спектра. Базовый, или мгновенный, квазиравновесный статистический оператор выбирается в форме, напоминающей каноническое распределение, и дается выражением

$$
\bar{\varrho}(t, 0)=\exp \left\{-\phi(t)-\sum_{\mathbf{k}} F_{\mathbf{k}}(t) \hat{n}_{\mathbf{k}}-\sum_{\mathbf{k}, \mathbf{Q} \neq 0} F_{\mathbf{k} \mathbf{Q}}(t) \hat{n}_{\mathbf{k} \mathbf{Q}}\right\}
$$

с функцией $\phi(t)$, обеспечивающей нормировку $\bar{\varrho}$ :

$$
\phi(t)=\ln \operatorname{Tr}\left\{\exp \left\{-\sum_{\mathbf{k}} F_{\mathbf{k}}(t) \hat{n}_{\mathbf{k}}-\sum_{\mathbf{k}, \mathbf{Q} \neq 0} F_{\mathbf{k} \mathbf{Q}}(t) \hat{n}_{\mathbf{k} \mathbf{Q}}\right\}\right\},
$$

и где мы полагаем

$$
\hat{n}_{\mathbf{k}}=c_{\mathbf{k}}^{\dagger} c_{\mathbf{k}}, \quad \hat{n}_{\mathbf{k} \mathbf{Q}}=c_{\mathbf{k}+\frac{1}{2} \mathbf{Q}}^{\dagger} c_{\mathbf{k}-\frac{1}{2} \mathbf{Q}}
$$

для электронов (спиновый индекс опущен или можно считать, что он включен в $\mathbf{k}$ ),

$$
\hat{n}_{\mathbf{k}}=h_{\mathbf{k}}^{\dagger} h_{\mathbf{k}}, \quad \hat{n}_{\mathbf{k Q}}=h_{\mathbf{k}+\frac{1}{2} \mathbf{Q}}^{\dagger} h_{\mathbf{k}-\frac{1}{2} \mathbf{Q}}
$$

для дырок,

$$
\hat{n}_{\mathbf{q}}=a_{\gamma_{\mu} \mathbf{q}}^{\dagger} a_{\gamma_{\mu} \mathbf{q}}, \quad \hat{n}_{\mathbf{q Q}}=a_{\gamma_{\mu} \mathbf{q}+\frac{1}{2} \mathbf{Q}}^{\dagger} a_{\gamma_{\mu} \mathbf{q}-\frac{1}{2} \mathbf{Q}}
$$

для фононов ветви $\gamma$, поляризации $\mu$ и моды $\mathbf{q}$ (которую мы предпочитаем обозначать q вместо $\mathbf{k}$ ). Мы не стали включать когерентные состояния и операторы пар, которые в общем случае не очень важны.

Обратим внимание на то, что функция $\phi(t)$ в выражении (20) играет роль логарифма неравновесной статистической суммы, скажем $\phi(t)=\ln \bar{Z}(t)$, и на то, что величины $F_{\mathbf{k}}(t)$ и $F_{\mathbf{k Q}}(t)$ являются неравновесными термодинамическими переменными, сопряженными каждому типу базисных динамических переменных, а именно $\hat{n}_{\mathbf{k}}$ и $\hat{n}_{\mathbf{k Q}}$ соответственно.

Основанная на ФНСА нелинейная кинетическая теория релаксационных процессов сводится в основном к усреднению по неравновесному ансамблю гейзенберговских (или гамильтоновых на классическом уровне) уравнений движения для динамических операторов рассматриваемых наблюдаемых, скажем для $\widehat{P}_{j}(\mathbf{r})$ с $j=$ $1,2, \ldots$ (фазовых функций в классической механике и эрмитовых операторов в квантовой механике), т. е.

$$
\frac{\partial}{\partial t} Q_{j}(\mathbf{r}, t)=\frac{\partial}{\partial t} \operatorname{Tr}\left\{\widehat{P}_{j}(\mathbf{r}) \varrho_{\varepsilon}(t)\right\}=\operatorname{Tr}\left\{\frac{1}{i \hbar}\left[\widehat{P}_{j}(\mathbf{r}), \widehat{H}\right] \varrho_{\varepsilon}(t)\right\}
$$


что является примером теоремы Эренфеста. Практические приложения этой кинетической теории описаны в работах [17]-[19], [24], [46]-[54], главным образом в [46].

Здесь мы кратко отметим, что особенно важен марковский предел кинетической теории, поскольку в широком круге проблем взаимодействия слабы и использование приближения низшего порядка (второго порядка по интенсивности взаимодействий) в уравнениях движения имеет большую практическую ценность. При помощи другого подхода Дэвис [59] показал, что на самом деле марковское приближение можно обосновать в пределе слабой связи (по взаимодействию).

Записанные в явном виде марковские уравнения в кинетической теории имеют вид

$$
\frac{\partial}{\partial t} Q_{j}(\mathbf{r}, t)=J_{j}^{(0)}(\mathbf{r}, t)+J_{j}^{(1)}(\mathbf{r}, t)+J_{j}^{(2)}(\mathbf{r}, t) .
$$

После того как в гамильтониане введено разложение $\widehat{H}=\widehat{H}_{0}+\widehat{H}^{\prime}$, где $\widehat{H}_{0}$ представляет собой кинетическую энергию, а $\widehat{H}^{\prime}$ содержит потенциальные энергии взаимодействий, имеем

$$
\begin{aligned}
& J_{j}^{(0)}(\mathbf{r}, t)=\operatorname{Tr}\left\{\frac{1}{i \hbar}\left[\widehat{P}_{j}(\mathbf{r}), \widehat{H}_{0}\right] \bar{\varrho}(t, 0) \times \varrho_{R}\right\}, \\
& J_{j}^{(1)}(\mathbf{r}, t)=\operatorname{Tr}\left\{\frac{1}{i \hbar}\left[\widehat{P}_{j}(\mathbf{r}), \widehat{H}^{\prime}\right] \varrho(t, 0) \times \varrho_{R}\right\},
\end{aligned}
$$

и $J_{j}^{(2)}(\mathbf{r}, t)={ }_{\mathrm{I}} J_{j}^{(2)}(\mathbf{r}, t)+{ }_{\mathrm{II}} J_{j}^{(2)}(\mathbf{r}, t)$ с

$$
\begin{gathered}
{ }_{\mathrm{I}} J_{j}^{(2)}(\mathbf{r}, t)=\frac{1}{(i \hbar)^{2}} \int_{-\infty}^{t} d t^{\prime} e^{\varepsilon\left(t^{\prime}-t\right)} \operatorname{Tr}\left\{\left[\widehat{H}^{\prime}\left(t^{\prime}-t\right)_{0},\left[\widehat{H}^{\prime}, \widehat{P}_{j}(\mathbf{r})\right]\right] \bar{\varrho}(t, 0) \times \varrho_{R}\right\}, \\
\mathrm{II}_{j} J_{j}^{(2)}(\mathbf{r}, t)=\frac{1}{i \hbar} \sum_{\mathbf{k}} \int_{-\infty}^{t} d t^{\prime} e^{\varepsilon\left(t^{\prime}-t\right)} \operatorname{Tr}\left\{\left[\widehat{H}^{\prime}, \widehat{P}_{k}(\mathbf{r})\right] \bar{\varrho}(t, 0) \times \varrho_{R}\right\} \frac{\delta J_{j}^{(1)}(\mathbf{r}, t)}{\delta Q_{k}(\mathbf{r}, t)},
\end{gathered}
$$

где $\bar{\varrho}$ - вспомогательный статистический оператор (20), а $\varrho_{R}-$ равновесное статистическое распределение для термостата. Напомним, что величины $J_{j}^{(0)}$ и $J_{j}^{(1)}$, которые согласно терминологии Мори [31] называются прецессионным и силовым членами, связаны с недиссипативным движением, в то время как диссипативные эффекты учитываются величинами $J_{j}^{(2)}$, которые можно назвать интегралами столкновений. Нижний индекс 0 указывает на эволюцию в представлении взаимодействия, символом $\delta$ означен функциональный дифференциал [60].

Приведенные здесь результаты позволяют утверждать, что ФНСА приводит к имеющей широкую область применимости обобщенной теории переноса, которая учитывает нелинейность, нелокальность в пространстве и времени (т.е. пространственные корреляции и эффекты памяти) и охватывает почти любые неравновесные ситуации в многочастичных системах. Обратим внимание на тот важный факт, что обобщенная процедура сглаживания по времени (в смысле Кирквуда), предполагаемая в формализме, проявляется в конечных уравнениях эволюции как затухающая память (опережающие решения отброшены). Поэтому макросостояние системы изменяется необратимым образом. Более того, если после приготовления неравновесного состояния на систему не действуют никакие источники возбуждения, но она находится в контакте с идеальными резервуарами, скажем энергии и частиц, 
то макросостояние системы стремится к конечному состоянию равновесия, которое описывается обычным большим каноническим распределением.

Мы дали общий обзор ФНСА и теперь приступаем к описанию СТНП, основанной на $Ф Н \mathrm{HCA}$.

\section{3. СТАТИСТИЧЕСКАЯ ТЕРМОДИНАМИКА НЕОБРАТИМЫХ ПРОЦЕССОВ}

Нелинейная неравновесная термодинамика и связанные с ней кинетическая и статистическая теории не достигли еще полностью удовлетворительного уровня, поэтому они являются объектом активного обсуждения и дискуссии (например, в одном частном аспекте, см. [61]). Первым и весьма успешным подходом (в области его применимости) к термодинамике необратимых процессов была классическая (иногда называемая линейной или онсагеровской) термодинамика, изложенная, например, в уже ставших классическими книгах [62], [63] и основанная главным образом на фундаментальных работах Онсагера [64], [65]. В 1950-е и 1960-е годы эта теория была развита за пределы линейного режима в так называемой обобщенной классической термодинамике Пригожиным и Брюссельской школой [66].

Чтобы охватить произвольные сильно неравновесные ситуации, делаются попытки заменить феноменологическую классическую термодинамику новыми подходами. Тисса [67] полагал, что существуют следующие четыре способа описания термодинамики.

1. Подход, основанный на двух фундаментальных законах термодинамики и правилах операций с циклами Карно. Иногда такой подход называют инженерным подходом или термодинамикой Клаузиуса-Кельвина.

2. Математический подход, основанный на дифференциальной геометрии и топологии вместо циклов Карно. Иногда этот подход называется термодинамикой Каратеодори-Борна.

3. Аксиоматическая точка зрения, состоящая в том, чтобы заменить циклы Карно и дифференциальную геометрию набором основных аксиом, которые охватывают два предыдущих подхода и их обобщения. Эту схему можно назвать термодинамикой Тиссы-Кэллена.

4. Статистико-механическая точка зрения, опирающаяся, конечно, на микроскопическую механику (на уровне молекул, атомов или частиц) и теорию вероятностей. Ее можно назвать термодинамикой Гиббса или статистической термодинамикой.

Сгруппировать все существующие попытки в рамках этой схемы - нелегкая задача. Мы отметим (и приносим извинения за то, что некоторые упущены) следующие несколько подходов: рациональная термодинамика, предложенная Трусделлом [68]; геометрически-топологический подход Ландсберга [7] - расширение и обобщение метода Каратеодори; то, что мы называем ортодоксальной термодинамикой необратимых процессов, предложенной Чан-Ю [9] и его последователями; расширенная термодинамика необратимых процессов, созданная и развитая несколькими авторами, значительно улучшенная и систематизированная Касас-Васкесом, Джоу и Лебоном из так называемой Каталонской школы термодинамики [69]-[73]; обобщенный кинетический подход, развитый Гарсиа-Колином и так называемой Мексиканской школой термодинамики [12]; волновой подход $\kappa$ термодинамике, принадлежащий 
Гьярмати [74]; так называемый универсальный подход Грмелы [75] и его последователей; холотропный подход Бернардеса [76]; информационная статистическая термодинамика (или информационно-теоретическая термодинамика), инициированная Хобсоном [8] и в систематизированном и расширенном виде подробно описанная в работах [6], [77]-[79]. Последняя представляет собой основанную на ФНСА СТНП, которую мы здесь кратко изложим.

Если многочастичная система находится в неравновесных условиях, характеризуемых неравновесным статистическим оператором в ФНСА, описанным в предыдущем разделе (см. (18)), это означает, что выбран базисный набор динамических переменных, обозначаемых в общем случае $\widehat{P}_{j}(\mathbf{r})$ (как уже отмечалось, это операторы, определяемые приведенными динамическими операторами из формул (1) и (3)). Тогда базовый (или мгновенный) квазиравновесный статистический оператор (20) имеет вид

$$
\bar{\varrho}(t, 0)=\exp \left\{-\phi(t)-\int d^{3} r \sum_{j} F_{j}(\mathbf{r}, t) \widehat{P}_{j}(\mathbf{r})\right\},
$$

где введен набор неравновесных термодинамических переменных $F_{j}(\mathbf{r}, t)$.

Средние от динамических величин $\widehat{P}_{j}(\mathbf{r})$ по неравновесному ансамблю, т. е.

$$
Q_{j}(\mathbf{r}, t)=\operatorname{Tr}\left\{\widehat{P}_{j}(\mathbf{r}) \varrho_{\varepsilon}(t)\right\}
$$

определяют в СТНП базисные переменные пространства неравновесных термодинамических состояний, эволюция которых описывается кинетическими уравнениями (26).

Говорят, что в рамках СТНП переменные $F_{j}(\mathbf{r}, t)$ являются сопряженными к базисным переменным $Q_{j}(\mathbf{r}, t)$ в том смысле, что

$$
Q_{j}(\mathbf{r}, t)=-\frac{\delta \phi(t)}{\delta F_{j}(\mathbf{r}, t)} \equiv-\frac{\delta \ln \bar{Z}(t)}{\delta F_{j}(\mathbf{r}, t)},
$$

с функцией $\phi(t) \equiv \ln \bar{Z}(t)$, которая, как отмечалось выше, играет роль логарифма неравновесной статистической суммы в ФНСА.

Далее опишем кратко некоторые аспекты СТНП; более детальное рассмотрение дается в работах [6], [77]-[79].

\section{4. ОБЩИЕ ТЕРМОДИНАМИЧЕСКИЕ СВОЙСТВА, ОПИСЫВАЕМЫЕ СТНП}

Энтропия Гиббса, непосредственное обобщение равновесной энтропии, а именно

$$
S_{\mathrm{G}}(t)=-\operatorname{Tr}\left\{\rho_{\varepsilon}(t) \ln \rho_{\varepsilon}(t)\right\},
$$

не подходит в качестве квазитермодинамической энтропии, поскольку она является интегралом движения, т. е. $d S_{\mathrm{G}}(t) / d t=0$ (следствие того, что $\rho_{\varepsilon}$ удовлетворяет модифицированному уравнению Лиувилля (18) с источником, который, напомним, стремится к нулю после того, как выполнена операция следа при вычислении средних). Это говорит о том, что $S_{\mathrm{G}}-$ мелкозернистая энтропия, которая сохраняет информацию. Эта информация задана в начальный момент $t_{0}$ приготовления системы; 


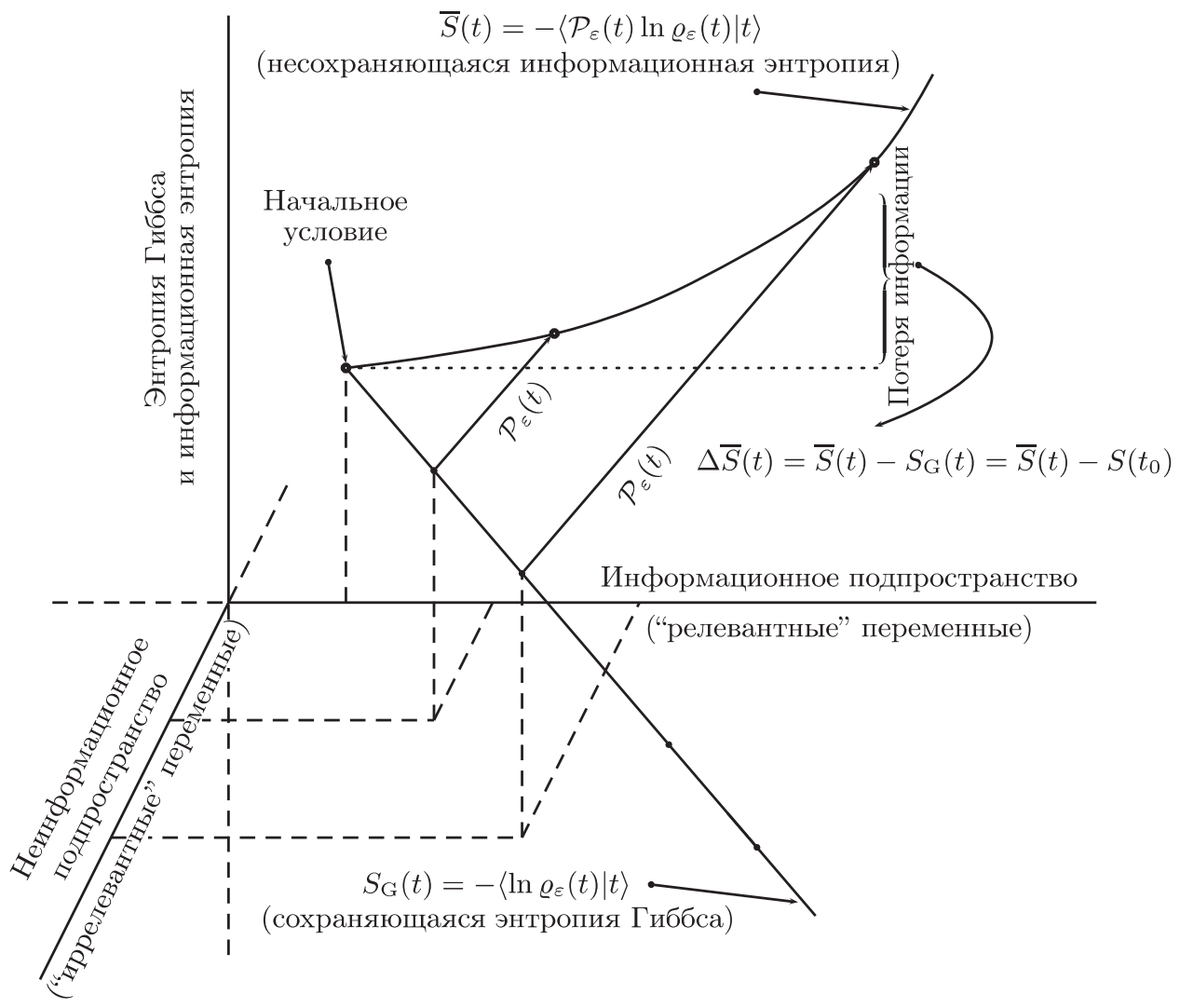

Рис. 1. Схема описания неравновесного диссипативного макроскопического состояния системы. Проецирование (зависящее от мгновенного макросостояния системы) вводит процедуру крупнозернистого усреднения, которая заключается в проецировании на подпространство "релевантных" переменных, связанных с информационными ограничениями в СТНП, т. е. с выбранными базисными переменными.

она выражается через начальные значения базисного набора переменных $\left\{Q_{j}\left(\mathbf{r}, t_{0}\right)\right\}$. Следовательно, для каждого последующего момента времени $t>t_{0}$ должна быть определена информационно-статистическая энтропия. Разность между этой энтропией $\bar{S}(t)$ и энтропией Гиббса (32) может служить мерой потери информации, когда макроскопическое состояние системы описывается сокращенным набором базисных переменных, а именно тем, что называется информационным подпространством "релевантных" переменных. Таким образом, с помощью рис. 1 определяется крупнозернистая СТНП-энтропия

$$
\bar{S}(t)=-\operatorname{Tr}\left\{\rho_{\varepsilon}(t) \mathcal{P}_{\varepsilon} \ln \rho_{\varepsilon}(t)\right\}=-\operatorname{Tr}\left\{\rho_{\varepsilon}(t) \ln \bar{\varrho}(t, 0)\right\},
$$

которая дается средним значением проекции логарифма мелкозернистого неравновесного статистического оператора, т. е. [17], [26]

$$
\mathcal{P}_{\varepsilon} \ln \rho_{\varepsilon}(t)=\ln \bar{\varrho}(t, 0),
$$


на подпространство, которое содержит базисный набор динамических переменных и называется информационным подпространством. Эту СТНП-энтропию (или информационно-статистическую энтропию) следует поставить в соответствие с энтропиями феноменологических теорий. Исходя из определения (33) можно воспроизвести как частные случаи соответствующие выражения из равновесной термодинамики, классической термодинамики необратимых процессов и расширенной термодинамики неравновесных процессов. Таким образом, СТНП можно считать далеко идущим обобщением существующих феноменологических подходов. Рассмотрим этот момент подробнее.

Используя выражение (29) для вспомогательного (крупнозернистого) статистического оператора $\bar{\varrho}(t, 0)$, мы можем записать СТНП-энтропию (33) как

$$
\bar{S}(t)=\int d^{3} r \bar{\eta}(\mathbf{r}, t)=\phi(t)+\int d^{3} r \sum_{j} F_{j}(\mathbf{r}, t) Q_{j}(\mathbf{r}, t),
$$

где $\bar{\eta}(\mathbf{r}, t)$ представляет собой локальную плотность информационной статистической энтропии. Более того, дифференциал СТНП-энтропии в пространственной точке $\mathbf{r}$ и в момент времени $t$ имеет вид пфаффовой формы (обобщенное соотношение Гиббса)

$$
d \bar{\eta}(\mathbf{r}, t)=\sum_{j} F_{j}(\mathbf{r}, t) d Q_{j}(\mathbf{r}, t) .
$$

Уравнения эволюции (25) или (26) базисных переменных, получаемые в формализме, в общем случае имеют вид уравнений баланса вида

$$
\frac{\partial}{\partial t} Q_{j}(\mathbf{r}, t)=-\operatorname{div} \mathbf{I}_{j}(\mathbf{r}, t)+\xi_{j}(\mathbf{r}, t)
$$

где $\mathbf{I}_{j}-$ поток переменной $Q_{j}$, а $\xi_{j}$ учитывает наличие источников и/или стоков для такой переменной (это упрощенное обозначение; напомним, что $Q_{j}$ могут быть скалярами, векторами и тензорами, т.е. скалярными величинами, их векторными потоками или тензорными потоками высших порядков [17], [80]-[85]). Используя уравнения (37), мы можем записать уравнение баланса для СТНП-энтропии:

$$
\frac{\partial}{\partial t} \bar{\eta}(\mathbf{r}, t)+\operatorname{div} \mathbf{I}_{\eta}(\mathbf{r}, t)=\sigma_{\eta}(\mathbf{r}, t)
$$

где $\mathbf{I}_{\eta}$ - поток СТНП-энтропии, который определяется как

$$
\mathbf{I}_{\eta}(\mathbf{r}, t)=\sum_{j} F_{j}(\mathbf{r}, t) \mathbf{I}_{j}(\mathbf{r}, t)
$$

а $\sigma_{\eta}$ включает источники и/или стоки вида

$$
\sigma_{\eta}(\mathbf{r}, t)=\sum_{j}\left\{\mathbf{I}_{j}(\mathbf{r}, t) \cdot \nabla F_{j}(\mathbf{r}, t)+F_{j}(\mathbf{r}, t) \xi_{j}(\mathbf{r}, t)\right\} .
$$

Уравнение (38) представляет собой наиболее общую форму уравнения баланса энтропии в СТНП. Хотя оно и напоминает уравнение, которое постулируется в расширенной термодинамике необратимых процессов, оно является гораздо более общим, 
так как $\mathbf{I}_{\eta}$ не обязательно включает только физические гидродинамические потоки и не делается никаких априорных предположений о знаке $\sigma_{\eta}$. Таким образом, установлено соответствие между феноменологическим и статистическим подходами к термодинамике необратимых процессов. Из (36) можно также увидеть, что

$$
F_{j}(\mathbf{r}, t)=\frac{\delta \bar{S}(t)}{\delta Q_{j}(\mathbf{r}, t)} .
$$

Это соотношение определяет неравновесные термодинамические переменные $F_{j}$ как коэффициенты в дифференциале информационно-статистической энтропии. Система равенств (41) связывает макропеременные $F_{j}$ с базисными макропеременными $Q_{j}$ (от которых зависит $\bar{S}$ ), поэтому равенства (41) можно рассматривать как уравнения состояния для неравновесных систем.

Напомним, что в феноменологической расширенной термодинамике необратимых процессов одним из основных постулатов является постулат о положительной определенности локального и мгновенного производства энтропии [69]-[73]. В СТНП производство энтропии получается путем дифференцирования по времени выражения (35) (ср. с (36)), в результате чего находим

$$
\bar{\sigma}(t)=\frac{d}{d t} \bar{S}(t)=\int d^{3} r \frac{\partial \bar{\eta}(\mathbf{r}, t)}{\partial t},
$$

где

$$
\frac{d \bar{\eta}(\mathbf{r}, t)}{d t} \equiv \bar{\sigma}(\mathbf{r}, t)=\sum_{j} F_{j}(\mathbf{r}, t) \frac{\partial}{\partial t} Q_{j}(\mathbf{r}, t),
$$

с производными по времени, которые следуют из кинетического уравнения (25) (или уравнения (26) в марковском приближении). В общем случае никакого определенного вывода о знаке $\bar{\sigma}(\mathbf{r}, t)$ сделать невозможно ввиду чрезвычайной сложности полученного выражения. Тем не менее можно доказать, что [6], [77]-[79]

$$
\bar{S}(t)-\bar{S}\left(t_{0}\right)=\bar{S}(t)-S_{\mathrm{G}}(t) \geqslant 0 .
$$

С помощью (42) это можно записать в виде соотношения

$$
\int_{t_{0}}^{t} d t^{\prime} \int d^{3} r \bar{\sigma}(\mathbf{r}, t) \geqslant 0
$$

(как и ранее, $t_{0}$ - начальное время приготовления системы), которое утверждает, что СТНП-энтропия не может убывать со временем. Мы назовем формулу (45) слабым приниипом неотрицательности производства информационной энтропии, а (44) можно рассматривать как выражение для $\mathcal{H}$-теоремъ в СТНП. Этот результат эквивалентен результату, полученному дель Рио и Гарсиа-Колином [86]; они интерпретировали заключительное неравенство следующим образом: последовательность наблюдений (в течение точно определенного интервала времени) над макроскопической системой, совершающей необратимый процесс, всегда приводит к потере информации в смысле Шеннона и Бриллюэна [87], [88]. Это иллюстрируется на рис. 1: разница между энтропиями двух типов (мелкозернистой и крупнозернистой), а именно среднее значение $\left[1-\mathcal{P}_{\varepsilon}\right] \ln \rho_{\varepsilon}(t)$, представляет собой рост 
информационно-статистической энтропии при необратимой эволюции системы (cp. (44) и (33)).

Можно показать также, что СТНП дает статистико-механические обобщения нескольким теоремам феноменологических термодинамик. Одной из них является закон Пригожина о минимальном производстве энтропии [89], который, напомним, устанавливает вариационный принцип, состоящий в том, что в линейном режиме около стационарного состояния системы вблизи равновесия скорость изменения со временем производства энтропии из-за внутренних релаксационных процессов отрицательна. В некотором смысле это принцип экономии: система стремится к состоянию, в котором производится настолько мало энтропии, насколько возможно. Другим достижением является обобщение термодинамического критерия эволюции Глансдорфа-Пригожина [66]: согласно ему эволюция системы в любых неравновесных условиях происходит таким образом, чтобы была отрицательна скорость изменения энтропии, связанная исключительно с изменением переменных $F_{j}$ в $(41)$, т. е. величина

$$
\frac{d_{F}}{d t} \sigma(t)=\sum_{j} \int d^{3} r \frac{\partial}{\partial t} F_{j}(\mathbf{r}, t) \frac{\partial}{\partial t} Q_{j}(\mathbf{r}, t) \leqslant 0 .
$$

Уравнение (46) содержит обобщение теоремы о минимальном производстве энтропии в линейном режиме около равновесия. Можно заметить, что этот критерий эволюции не является вариационным принципом, поскольку он не включает точный дифференциал, как в случае линейного режима около равновесия, когда производство энтропии, связанное с релаксационными процессами в стационарном состоянии, может играть роль термодинамического потенциала. По-видимому, невозможно определить подходящие термодинамические потенциалы, чтобы описать состояния произвольных неравновесных систем, в отличие от равновесного случая и уже упомянутого линейного режима, которые допускают такой подход. Применение ФНСА к изучению термодинамики упрощенной модели неравновесного полупроводника, которое иллюстрирует два только что описанных принципа, излагается в работе [90].

Информационная энтропия в СТНП удовлетворяет также обобщенному соотношению Клаузиуса. Действительно, рассмотрим изменение информационной энтропии вследствие изменений внешних ограничений, наложенных на систему. Обозначим через $\lambda_{l}, l=1,2, \ldots, s$, набор параметров, которые характеризуют такие ограничения (например, объем, число частиц, внешние поля и т.д.). Если ввести их инфинитезимальные приращения, скажем $d \lambda_{l}$, то соответствующее изменение информационной энтропии (для произвольных $\left\{Q_{j}\right\}$ и $\left\{F_{j}\right\}$ ) дается формулой

$$
d \bar{S}(t)=\int d^{3} r \sum_{j} F_{j}(\mathbf{r}, t) \delta Q_{j}(\mathbf{r}, t),
$$

где $\delta Q_{j}-$ неточные дифференииаль

$$
\delta Q_{j}(\mathbf{r}, t)=d Q_{j}(\mathbf{r}, t)-\left\langle d \widehat{P}_{j}(\mathbf{r}) \mid t\right\rangle
$$

с $\left\langle d \widehat{P}_{j}(\mathbf{r}) \mid t\right\rangle=\operatorname{Tr}\left\{d \widehat{P}_{j} \bar{\varrho}(t, 0)\right\}$. В этих выражениях неточный дифференциал есть разность между точным дифференциалом

$$
d Q_{j}(\mathbf{r}, t)=d \operatorname{Tr}\left\{\widehat{P}_{j}(\mathbf{r}) \bar{\varrho}(t, 0)\right\}=\sum_{l=1}^{s} \frac{\partial Q_{j}(\mathbf{r}, t)}{\partial \lambda_{l}} d \lambda_{l}
$$


и величиной

$$
\left\langle d \widehat{P}_{j}(\mathbf{r}) \mid t\right\rangle=\operatorname{Tr}\left\{\sum_{l=1}^{s} \frac{\partial \widehat{P}_{j}(\mathbf{r})}{\partial \lambda_{l}} d \lambda_{l} \bar{\varrho}(t, 0)\right\}
$$

которая есть среднее значение изменения соответствующей динамической переменной из-за изменения управляющих параметров.

Равенство (47) показывает, что неравновесные термодинамические переменные $F_{j}$ являются интегрирующими множителями для неточных дифференциалов $\delta Q_{j}$.

Выберем в качестве одной из переменных $Q_{j}$ плотность энергии, например $Q_{1}(\mathbf{r}, t)=\epsilon(\mathbf{r}, t)$, и по аналогии с равновесием определим интенсивную неравновесную термодинамическую переменную, которую назовем квазитемпературой, или, лучше сказать, обратную к ней величину (в единицах $k_{\mathrm{B}}$ ):

$$
\Theta^{-1}(\mathbf{r}, t)=\frac{\delta \bar{S}(t)}{\delta \epsilon(\mathbf{r}, t)}
$$

После дополнительного переопределения неравновесных термодинамических переменных

$$
F_{j}(\mathbf{r}, t)=\Theta^{-1}(\mathbf{r}, t) \mathcal{F}_{j}(\mathbf{r}, t)
$$

использование равенства (36) позволяет нам ввести разновидность обобщенного, зависящего от пространственных координат соотношения Клаузиуса для системы в произвольных неравновесных условиях, а именно

$$
\bar{\eta}(\mathbf{r}, t)-\bar{\eta}\left(\mathbf{r}, t_{0}\right)=\int_{t_{0}}^{t} d t^{\prime} \frac{\partial \bar{\eta}\left(\mathbf{r}, t^{\prime}\right)}{\partial t^{\prime}}=\int_{t_{0}}^{t} d t^{\prime} \Theta^{-1}\left(\mathbf{r}, t^{\prime}\right) \delta \dot{q}\left(\mathbf{r}, t^{\prime}\right),
$$

где мы ввели неточный дифференциал обобщенной тепловой функции $q(\mathbf{r}, t)$ :

$$
\delta q\left(\mathbf{r}, t^{\prime}\right)=\delta \epsilon\left(\mathbf{r}, t^{\prime}\right)+\sum_{j=2} \mathcal{F}_{j}\left(\mathbf{r}, t^{\prime}\right) \delta Q_{j}\left(\mathbf{r}, t^{\prime}\right) .
$$

В (53) интегрирование по времени следует понимать как интегрирование вдоль траектории эволюции системы, описываемой кинетическими уравнениями (26).

Далее, используя переопределение переменных (52), можно заметить, что зависящее от пространственных координат и времени обобщенное соотношение Гиббса (36) принимает вид

$$
\Theta(\mathbf{r}, t) d \eta(\mathbf{r}, t)=d \epsilon(\mathbf{r}, t)+\sum_{j=2} \mathcal{F}_{j}(\mathbf{r}, t) d Q_{j}(\mathbf{r}, t),
$$

где в левой части явно выписан член с квазитемпературой $\Theta$ (подчеркнем, что в (54) символом $\delta Q$ обозначен неточный дифференциал, возникающий из-за изменения ограничений, в то время как в (55) входят дифференциалы $d Q$ переменных, от которых зависит функция $\bar{S})$.

Интегрируя равенство (53) по пространству и учитывая все результаты, которые привели к $\mathcal{H}$-теореме $(44)$, можно записать

$$
\bar{S}(t)-\bar{S}\left(t_{0}\right)=\bar{S}(t)-S_{\mathrm{G}}(t)=\int d^{3} r \int_{t_{0}}^{t} d t^{\prime} \Theta^{-1}\left(\mathbf{r}, t^{\prime}\right) \delta \dot{q}\left(\mathbf{r}, t^{\prime}\right) \geqslant 0
$$


Вид неравенства (56) подсказывает его “вольную интерпретацию” как результат применения своеобразного принципа Карно для произвольных неравновесных систем, смысл которого состоит в том, что вклад в подынтегральное выражение представляет собой обратимый обмен некоторой величиной (типа теплоты) между системой и псевдорезервуаром с локальной мгновенной температурой $\Theta(\mathbf{r}, t)$. Некоторые соображения по поводу принципа Карно и его связи с СТНП как общего принципа логических рассуждений были выдвинуты Джейнсом [91]. Он описал эволюцию принципа Карно от вывода Кельвина о том, что этот принцип определяет универсальную шкалу температур, открытия Клаузиуса, что этот принцип предполагает существование энтропии, догадки Гиббса о логическом статусе этого принципа и интерпретации Больцманом энтропии в терминах фазового объема к общему формализму статистической механики.

Рассмотрим теперь вопрос о флуктуациях и соотношениях Максвелла в неравновесных условиях. Как уже отмечалось, в СТНП среднее значение любой динамической величины из базисного набора (для простоты будем здесь использовать классический уровень описания) дается формулой

$$
Q_{j}(t)=\int d \Gamma \widehat{P}_{j}(\Gamma) \bar{\varrho}(\Gamma \mid t, 0)=-\frac{\delta \phi(t)}{\delta F_{j}(t)}
$$

т. е. оно равно (с обратным знаком) функциональной производной производящего функционала $\phi$ по соответствующей термодинамической переменной $F_{j}(t)$ (напомним, что этот функционал можно связать с неравновесной статистической суммой с помощью выражения $\phi(t)=\ln \bar{Z}(t))$. Далее, из прямого вычисления следует, что

$$
\frac{\delta^{2} \phi(t)}{\delta F_{j}(t) \delta F_{k}(t)}=-\frac{\delta Q_{j}(t)}{\delta F_{k}(t)}=\int d \Gamma \Delta \widehat{P}_{j}(\Gamma) \Delta \widehat{P}_{k}(\Gamma) \bar{\varrho}(\Gamma \mid t, 0)=\mathcal{C}_{j k}(t)
$$

где

$$
\Delta \widehat{P}_{j}(\Gamma)=\widehat{P}_{j}(\Gamma)-\int d \Gamma \widehat{P}_{j}(\Gamma) \bar{\varrho}(\Gamma \mid t, 0)=\widehat{P}_{j}(\Gamma)-Q_{j}(t)
$$

и соотношение (58) определяет матрицу корреляций $\widehat{\mathcal{C}}(t)$. Диагональные элементы $\widehat{\mathcal{C}}$ суть среднеквадратичные отклонения, или флуктуации, величин $\widehat{P}_{j}(\Gamma)$, а именно

$$
\begin{aligned}
C_{j j}(t) & \left.=\int d \Gamma \Delta \widehat{P}_{j}(\Gamma)\right]^{2} \bar{\varrho}(\Gamma \mid t, 0)= \\
& =\int d \Gamma\left[\widehat{P}_{j}(\Gamma)-Q_{j}(t)\right]^{2} \bar{\varrho}(\Gamma \mid t, 0) \equiv \Delta^{2} Q_{j}(t),
\end{aligned}
$$

и матрица является симметричной, т. е.

$$
\mathcal{C}_{j k}(t)=\frac{\delta^{2} \phi(t)}{\delta F_{j}(t) \delta F_{k}(t)}=\frac{\delta^{2} \phi(t)}{\delta F_{k}(t) \delta F_{j}(t)}=\mathcal{C}_{k j}(t),
$$

что в СТНП имеет сходство с равновесными соотношениями Максвелла. 
Введем теперь шкалу для информационной энтропии и неравновесных термодинамических переменных, используя постоянную Больцмана $k_{\mathrm{B}}$, т. е. положим

$$
S(t)=k_{\mathrm{B}} \bar{S}(t), \quad \mathcal{F}_{j}(t)=k_{\mathrm{B}} F_{j}(t)
$$

Тогда вследствие (41) имеем

$$
\mathcal{F}_{j}(t)=\frac{\delta S(t)}{\delta Q_{j}(t)}
$$

Кроме того, находим, что

$$
\frac{\delta^{2} S(t)}{\delta Q_{j}(t) \delta Q_{k}(t)}=\frac{\delta \mathcal{F}_{j}(t)}{\delta Q_{k}(t)}=\frac{\delta \mathcal{F}_{k}(t)}{\delta Q_{j}(t)}=-k_{\mathrm{B}} \mathcal{C}_{j k}^{(-1)}(t)
$$

т. е. функциональные производные второго порядка СТНП-энтропии пропорциональны (с обратным знаком) элементам $\mathcal{C}_{j k}^{(-1)}(t)$ матрицы, обратной к матрице корреляций $\mathcal{C}$. Далее, флуктуация СТНП-энтропии дается формулой

$$
\Delta^{2} S(t)=\sum_{j, k} \frac{\delta S(t)}{\delta Q_{j}(t)} \frac{\delta S(t)}{\delta Q_{k}(t)} \mathcal{C}_{j k}(t)=\sum_{j, k} \mathcal{C}_{j k}(t) \mathcal{F}_{j}(t) \mathcal{F}_{k}(t)
$$

а флуктуации интенсивных переменных $\mathcal{F}_{j}$ равны

$$
\Delta^{2} \mathcal{F}_{j}(t)=\sum_{k, l} \frac{\delta \mathcal{F}_{j}(t)}{\delta Q_{k}(t)} \frac{\delta \mathcal{F}_{j}(t)}{\delta Q_{l}(t)} \mathcal{C}_{j k}(t)=k_{\mathrm{B}}^{2} \sum_{k, l} \mathcal{C}_{j k}^{(-1)}(t) \mathcal{C}_{j l}^{(-1)}(t) \mathcal{C}_{k l}(t)=k_{\mathrm{B}}^{2} \mathcal{C}_{j j}^{(-1)}(t),
$$

поэтому получаем

$$
\Delta^{2} Q_{j}(t) \Delta^{2} \mathcal{F}_{j}(t)=k_{\mathrm{B}}^{2} \mathcal{C}_{j j}(t) \mathcal{C}_{j j}^{(-1)}(t)=k_{\mathrm{B}}^{2} G_{j j}(t),
$$

где

$$
G_{j j}(t)=\mathcal{C}_{j j}(t) \mathcal{C}_{j j}^{(-1)}(t)
$$

а затем находим

$$
\left[\Delta^{2} Q_{j}(t)\right]^{1 / 2}\left[\Delta^{2} \mathcal{F}_{j}(t)\right]^{1 / 2}=k_{\mathrm{B}} G_{j j}^{1 / 2}(t) .
$$

Вспоминая, что величины $\mathcal{C}_{j k}^{(-1)}$ являются матричными элементами матрицы, обратной к матрице корреляций, приходим к заключению, что если переменные не коррелированы, то $G_{j j}(t)=1$. Равенство (68) похоже на приниип неопределенно$c m u$, связывающий переменные $Q_{j}(t)$ и $\mathcal{F}_{j}(t)$, которые термодинамически сопряжены в смысле (41), причем постоянная Больцмана является атомистическим параметром, играющим роль, напоминающую роль кванта действия в механике. Это позволяет связать результаты СТНП с идеей дополнительности между микроскопическим и макроскопическим описаниями многочастичных систем, выдвинутой Розенфельдом и Пригожиным [92], [93]; этот момент обсуждается в [94] и более подробно рассмотрен в разделе 5 . 
Кроме того, можно вывести соотношение, напоминающее соотношение Больцмана между СТНП-энтропией и числом механических состояний, совместимых с неравновесным состоянием системы. Точно так же, как и в случае равновесия, оказывается, что отношение корня из среднеквадратичного отклонения данной величины к ее среднему значению имеет порядок обратного квадратного корня из числа частиц, т. е.

$$
\frac{\left[\Delta^{2} Q_{j}(t)\right]^{1 / 2}}{Q_{j}(t)} \sim N^{-1 / 2} .
$$

Следовательно, опять совершенно аналогично случаю равновесия, число состояний, дающих вклад в величину $P_{j}$ с заданным средним значением, чрезвычайно велико (для точного доказательства следует обратиться к методу быстрейшего спуска). По этой причине мы можем записать

$$
\begin{aligned}
\phi(t) & =\ln \int d \Gamma \exp \left\{-\sum_{j=1}^{n} F_{j}(t) \widehat{P}_{j}(\Gamma)\right\} \simeq \\
& \simeq \ln \left[\exp \left\{-\sum_{j=1}^{n} F_{j}(t) Q_{j}(t)\right\} \int_{\mathcal{M}(t)} d \Gamma\right]= \\
& =-\sum_{j=1}^{n} F_{j}(t) Q_{j}(t)+\ln \int_{\mathcal{M}(t)} d \Gamma
\end{aligned}
$$

где интегрирование ведется по многообразию $\mathcal{M}(t)$ в фазовом пространстве, образованному фазовыми точками $\Gamma \in \mathcal{M}(t)$ такими, что

$$
\mathcal{M}(t): Q_{j}(t) \leqslant \widehat{P}_{j}(\Gamma) \leqslant Q_{j}(t)+\Delta Q_{j}(t)
$$

где $\Delta Q_{j}(t)$ имеет порядок $\mathcal{C}_{j j}^{1 / 2}(t)$. Таким образом, из соотношений $(70)$ и $(62)$ следует, что

$$
\begin{aligned}
S(t) & =k_{\mathrm{B}} \phi(t)+\sum_{j=1}^{n} \mathcal{F}_{j}(t) Q_{j}(t) \simeq \\
& \simeq-\sum_{j=1}^{n} \mathcal{F}_{j}(t) Q_{j}(t)+k_{\mathrm{B}} \ln \int_{\mathcal{M}(t)} d \Gamma+\sum_{j=1}^{n} \mathcal{F}_{j}(t) Q_{j}(t),
\end{aligned}
$$

и затем

$$
S(t)=k_{\mathrm{B}} \ln W\left\{Q_{j}(t)\right\},
$$

где

$$
W\left\{Q_{j}(t)\right\}=\text { протяженность } \mathcal{M}(t) \equiv \operatorname{ext}\{\mathcal{M}(t)\},
$$

здесь "протяженность" означает размер гиперобъема в фазовом пространстве, занимаемого фазовыми точками, которые принадлежат $\mathcal{M}(t)$. Напомним, что это приближенный результат с ошибкой порядка обратного квадратного корня из числа степеней свободы системы и поэтому точный только в термодинамическом пределе. 
Соотношение (73) в СТНП представляет собой эквивалент выражения Больцмана для термодинамической энтропии через логарифм числа микросостояний, совместимых с макроскопическими ограничениями, наложенными на систему. Следует заметить, что в ФНСА они даются так называемым информационным набором, т. е. набором $\left\{Q_{j}(t)\right\}$, состоящим из средних значений механических переменных $\widehat{P}_{j}(t)$. Кроме того, они однозначно связаны с набором интенсивных термодинамических переменных, которые также полностью описывают макроскопическое состояние системы, т. е. с набором $\left\{\mathcal{F}_{j}(t)=k_{\mathrm{B}} F_{j}(t)\right\}$.

Выражение (73) на квантовом уровне описания выводится аналогично и имеет вид

$$
W\left\{Q_{j}(t)\right\}=\sum_{\tilde{n} \in \mathcal{M}(t)} 1=\text { число состояний в } \mathcal{M}(t) ;
$$

$\tilde{n}$ - набор квантовых чисел, характеризующих квантово-механическое состояние системы, а $\mathcal{M}$ содержит набор состояний $|\tilde{n}\rangle$ таких, что

$$
\mathcal{M}(t): Q_{j}(t) \leqslant\left\langle\tilde{n}\left|\widehat{P}_{j}\right| \tilde{n}\right\rangle \leqslant Q_{j}(t)+\Delta Q_{j}(t),
$$

где мы использовали обычное скобочное обозначение для матричных элементов между квантовыми состояниями.

На основе приведенных выше результатов можно получить альтернативную форму $\mathcal{H}$-теоремы:

$$
\bar{S}(t)-\bar{S}\left(t_{0}\right)=k_{\mathrm{B}} \ln \frac{\operatorname{ext}\{\mathcal{M}(t)\}}{\operatorname{ext}\left\{\mathcal{M}\left(t_{0}\right)\right\}} \geqslant 0,
$$

где $\operatorname{ext}\{\mathcal{M}(t)\}$ означает протяженность многообразия $\mathcal{M}(t)$ в классическом подходе и число состояний в квантовом подходе, фиксированные информационными ограничениями. Очевидно, соотношение (77) говорит нам, что протяженность многообразия $\mathcal{M}(t)$ возрастает со временем, что можно интерпретировать следующим образом. Рассмотрим замкнутую систему, которая вначале находится в сильно неравновесном состоянии и из него эволюционирует к конечному равновесию, причем эволюция описывается кинетическими уравнениями (26). Как было отмечено Боголюбовым, с течением времени корреляции затухают и для описания макроскопического состояния требуется все меньше переменных. В СТНП это соответствует уменьшению информационного пространства, что приводит, конечно, к уменьшению информации, переходу к ситуации с меньшими ограничениями и, как следствие, к росту протяженности многообразия $\mathcal{M}(t)$ и росту информащионной энтропии. Цитируя Джейнса, скажем, что именно это свойство энтропии - быть мерой нашей информации о микросостоянии, отраженной в значениях макроскопических термодинамических переменных, - делает теорию информации столь мощным средством обобщения равновесных ансамблей Гиббса на неравновесный случай. Этого обобщения никогда не удалось бы достичь, если считать, что энтропия является, подобно энергии, физическим свойством микросостояния [95]. Следуя также Джейнсу, можно сказать, что $W(t)$ измеряет степень контроля микросостояния экпериментатором, когда единственными параметрами, которыми может манипулировать экспериментатор, являются обычные макроскопические параметры. В момент $t$, когда производится измерение, состояние характеризуется набором $\left\{Q_{j}(t)\right\}$, а соответствующий фазовый объем равен $W(t)$ и содержит все возможные способы, которыми может быть 
реализовано макросостояние. Но, поскольку эксперимент должен быть воспроизводимым, область с объемом $W(t)$ должна содержать, по крайней мере, фазовые точки из области с объемом $W\left(t_{0}\right)$, и тогда $W(t) \geqslant W\left(t_{0}\right)$. Так как фазовый объем сохраняется в микродинамической эволюции, фундаментальным требованием к любому воспроизводимому процессу является то, что фазовый объем $W(t)$, совместимый с конечным состоянием, не может быть меньше фазового объема $W\left(t_{0}\right)$, который характеризует нашу способность воспроизвести начальное состояние [96].

Наконец, можно вывести обобщение критерия (не)устойчивости ГлансдорфаПригожина [66]. Согласно этому критерию, стационарное состояние открытой системы достаточно далеко от равновесия становится неустойчивым, когда меняет знак так называемая функция избыточного производства энтропии, а именно разность между внутренним производством энтропии в состоянии, немного отличном от стационарного состояния, и производством энтропии в самом стационарном состоянии. В этом состоит физический смысл метода линейного математического анализа устойчивости. Для того чтобы возникла неустойчивость, макроскопическое состояние системы, которое изменяется согласно нелинейным уравнениям эволюции, должно быть достаточно далеким от равновесия, так как в линейном режиме около равновесия теорема о минимальном производстве энтропии гарантирует его устойчивость. Следовательно, для возникновения нестабильностей в нелинейном режиме не обязаны существовать термодинамический потенциал и соответствующий вариационный принцип, как это уже отмечалось выше. Стабильность линейного режима является следствием неизбежного затухания флуктуаций, которое всегда сопровождается уменьшением порядка. С другой стороны, в нелинейном режиме в точке неустойчивости флуктуации могут нарастать и затем стабилизироваться в новом состоянии. Это является результатом “конкуренции” между эффектами диссипации и эффектами положительной обратной связи, возникающими благодаря нелинейным членам в уравнениях эволюции. Точка неустойчивости, характеризуемая критическим значением внешних сил, определяет то, что называется точкой бифуркации решений связанной системы нелинейных уравнений эволюции; в этой точке из прежнего, ставшего теперь неустойчивым, решения возникает новое устойчивое решение. Происходит переход между двумя стационарными состояниями, причем в новом устойчивом состоянии на макроскопической шкале возникает своего рода дальний порядок, или самоорганизация, включающая диссипативные структурь Пригожина, которые уже упоминались в разделе 1 в связи с теорией динамических систем со сложным поведением [5], [97], [98]. Таким образом, возникает связь СТНП, основанной на ФНСТ, с вопросами диссипации, порядка и сложности реальных систем.

Далее, обратим внимание на трудности, связанные с определениями неравновесной энтропии и неравновесной температуры (а также неравновесного химического потенциала, давления и т. д.). В случае энтропии вопрос заключается в том, может ли она быть подходящей функцией состояния, зависящей от подходяще выбранного набора базисных макропеременных. Как мы видели, эта проблема решается в условиях равновесия и локального равновесия, но не решается в общем случае, поскольку отсутствует полностью удовлетворительная теория, позволяющая сделать однозначный и хорошо определенный выбор. 
Вопрос об определении и измерении неравновесной квазитемпературы - очень интересная тема. Величина, которая может быть определена в расширенной термодинамике необратимых процессов и СТНП по аналогии с равновесием и названа квазитемпературой $\Theta(\mathbf{r}, t)$, дается выражением

$$
\Theta_{l}^{-1}(\mathbf{r}, t)=k_{\mathrm{B}} \frac{\delta \bar{S}(t)}{\delta \epsilon_{l}(\mathbf{r}, t)},
$$

где $\epsilon_{l}(\mathbf{r}, t)$ - макропеременная плотности энергии, а индекс $l$ означает дополнительные сведения о различных подсистемах в данном веществе. Важно иметь в виду, что, в отличие от равновесной термодинамики и классической термодинамики необратимых процессов, где температура зависит только от энергии и числа частиц, в СТНП она зависит от всех базисных макропеременных, включая потоки. Этот вопрос рассматривался в рамках феноменологической расширенной термодинамики необратимых процессов, и был предложен возможный, хотя и спорный, эксперимент для измерения [99]; случай фотоинжектированной плазмы в полупроводниках под воздействием постоянного электрического поля рассматривался в работе [100]. Конечно, важным является следующий вопрос: можно ли измерить неравновесную температуру (78)? Это необходимо, чтобы иметь последовательную и приемлемую теорию, поскольку температура является основной интенсивной макропеременной, связанной с макроскопическим описанием системы. Показано [101], что функции отклика системы в эксперименте зависят от базисных интенсивных переменных, поэтому эксперимент дает возможность измерить квазитемпературу (78). Подробное рассмотрение и сравнение с экспериментом приводятся в работе [102] (примеры из неравновесной физики полупроводников см. в [103]-[106]).

\section{5. ДОПОЛНИТЕЛЬНОСТЬ ОПИСАНИЙ}

По вопросу о связи и взаимодействии между микроскопическим и макроскопическим уровнями описания материи, т. е. между механикой и термодинамикой, Розенфельд [92], [93] заявлял, что в данном случае имеется некая логическая связь, к которой применим термин дополнительность. Такого же мнения придерживались Бор [107] и Пригожин [108]. По словам Розенфельда, эту связь следует характеризовать как взаимную исключительность двух описаний: условия, допускающие полностью микроскопическое описание системы, исключают возможность применения к системе любого из типичных термодинамических понятий, и, наоборот, макроскопическое описание требует условий наблюдения, при которых механические параметры не поддаются нашему контролю.

Эти идеи были проанализированы в контексте СТНП в работе [94], где было показано, что в ней имеется своего рода соотношение неопределенностей, включающее базисные макроскопические переменные, скажем $Q_{j}(t)($ cр. $(30)$ и $(31))$, и сопряженные им термодинамические переменные $F_{j}(t)($ cp. $(66))$, а именно

$$
\Delta^{2} Q_{j}(t) \Delta^{2} \mathcal{F}_{j}(t)=k_{\mathrm{B}}^{2}
$$

где $\mathcal{F}_{j}(t)=k_{\mathrm{B}} F_{j}(t)$, а величины $($ ср. $(30))$

$$
\Delta^{2} Q_{j}(t)=\operatorname{Tr}\left\{\left[\widehat{P}_{j}-Q_{j}(t)\right]^{2} \varrho_{\varepsilon}(t)\right\}
$$


и

$$
\Delta^{2} \mathcal{F}_{j}(t)=\sum_{k, \ell} \frac{\delta^{2} \phi(t)}{\delta F_{j}(t) \delta F_{k}(t)} \frac{\delta \mathcal{F}_{j}(t)}{Q_{k}(t)} \frac{\delta \mathcal{F}_{j}(t)}{Q_{\ell}(t)}
$$

являются среднеквадратичными отклонениями $Q_{j}$ и $\mathcal{F}_{j}$.

Можно считать, что равенство (79) выражает некоторый вид соотношения неопределенностей, или, правильнее, является мерой неполноты описания. Одновременное определение информационного содержания решения уравнений эволюции макросостояния и точное определение микросостояния (точки в фазовом пространстве или квантового состояния) невозможны. этот факт выражается наличием постоянной Больцмана: приводились аргументы [109] в пользу того, что величина $k_{\text {в }}$ учитывает влияние макроскопического эксперимента на микроскопический уровень описания. При этом теплота и работа рассматриваются как внутренние свойства вещества, а поток тепла - как движение “тепловых зарядов" под действием градиента

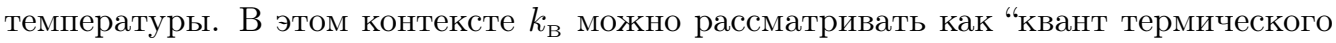
заряда", а именно как наименьшее количество тепла, которое может быть перенесено при единичном градиенте температуры. Это дало бы единицу неопределенности описания механического состояния при заданной сокращенной макроскопической характеристике системы. Этот момент отмечал также Тисса [67]. Следовательно, можно предположить, что так же, как постоянная Планка, характеризует неразделимость взаимодействия квантовой системы и измерительного устройства, постоянная Больцмана характеризует неразделимость микроскопического и макроскопического описаний [110]. В данном случае снова видно, что имеется своеобразная логическая связь, к которой применим, как расширение идей Бора, термин дополнительность, и можно предположить, что постоянная Больцмана играет роль элементарного кванта тепла и ответственна за неустранимый дуализм описаний.

\section{6. ИНФОРМАЦИЯ И СТАТИСТИЧЕСКАЯ ТЕРМОДИНАМИКА}

Ландауэр [111] писал о том, что “термодинамика возникла в XIX веке из попыток понять пределы эффективности тепловых машин, предугадывая, в известной степени, свойства всех дальнейших изобретений. После Второй мировой войны Клод Шеннон анализировал пределы эффективности каналов связи. Неудивительно, что вскоре после появления моделей цифровых вычислений похожие вопросы возникли и в этой области. Было нетрудно сопоставить логический вентиль со степенью свободы, затем связать с ним энергию $k_{\mathrm{B}} T$ и предположить, что именно такая энергия должна рассеиваться на каждом шаге".

Начиная с новаторской работы Шеннона [87] обсуждалась возможная связь между энтропией и информацией. Все началось с определения энтропии Шеннона $-\sum_{n} p_{n} \ln p_{n}$, где $p_{n}$ - безразмерная функция распределения некоторой подходящей переменной для системы в заданном состоянии $n$. Термин "энтропия" вводит в заблуждение; более правильным был бы термин “мера неопределенности информации" (или "теоретико-информационная энтропия"). Существует очень много подобных "теоретико-информационных энтропий”, что является следствием так называемых "скрытых ограничений" в описании состояния системы. Роль этих "энтропий" заключается в выводе распределения плотности вероятности в решаемой задаче с использованием вариационного принципа, который обычно называется 
формализмом максимума (информационной) энтропии. Все эти вопросы описаны в следующем разделе.

Наконец, касательно модного в настоящее время вопроса инноваций (в основной триаде: наука, технология, инновация) отмечалось [112], что инновация, направленная на улучшение каких-то характеристик, которые имели бы важное преимущество на рынке, не всегда может быть реализована, так как это недостижимо в рамках существующей технической инфраструктуры или запрещено законами природы. Например, законы термодинамики устанавливают абсолютный предел на расход топлива самолетами и автомобилями.

Сообщалось [113], что почти 25 лет назад Ландауэр [111] высказал предположение, что информация является физическим понятием. Она хранится в физических системах, передается физическим образом и обрабатывается физическими устройствами. Поэтому он пришел к выводу, что энтропия должна подчиняться физическим законам, в частности законам термодинамики.

Считается, что первый намек на связь между информацией и термодинамикой восходит к Максвеллу, который в 1867 году ввел своего ныне знаменитого демона. Другие соображения принадлежат Сцилларду [114], уже упомянутому Ландауэру [111] и Бенетту [115]. Лутц и Килиберто [113] считали, что термодинамика информации способна дать новые идеи в физике, химии и биологии и что ее даже можно распространить на область квантовой механики, где она привела бы к созданию законченной термодинамики квантовой информации.

\section{7. ЗАКЛЮЧИТЕЛЬНЫЕ ЗАМЕЧАНИЯ}

В данной статье мы кратко и неполно описали, как в рамках ФНСА представить феноменологическую термодинамику необратимых процессов в форме СТНП.

В разделе 2 кратко описан ФНСА, а в разделе 3 представлено построение СТНП в рамках $Ф Н С А$. Далее в разделе 4 следует изложение общих термодинамических свойств, которые описываются СТНП, а именно: определение соответствующей информационно-статистической энтропии и ее производства, неравновесные уравнения состояния, обобщенная $\mathcal{H}$-теорема, критерии эволюции и (не)устойчивости макроскопического состояния системы, обобщенные соотношения Клаузиуса, флуктуации и соотношения типа соотношений Максвелла и выражение для информационно-статистической энтропии типа выражения Больцмана.

Что касается СТНП-энтропии, можно добавить некоторые соображения. По поводу энтропии Скляр [116] отмечает, что понятие энтропии - наиболее термодинамическое понятие, а Брикмонт [117]--[119] заметил, что по отношению к энтропии существует некоторая таинственность. Как известно, это понятие первоначально возникло из доказательства Клаузиуса, что существование энтропии как функции состояния является фундаментальным следствием второго закона. Следуя Скляру, добавим, что благодаря абстрактности энтропии, ее важной роли в теории, а также отсутствию связи энтропии с непосредственным чувственным восприятием и простыми измерениями (как у температуры) неудивительно, что при поиске статистико-механического аналога неравновесной термодинамической энтропии у нас для этого практически нет указаний в остальной теории. Вопрос о том, что выбрать в качестве замены для энтропии, является открытым. Таким образом, возникает широкий выбор “энтропий”; каждая хорошо работает для тех целей, для 
которых она была введена. Мейкснер [120]-[122] спрашивает: “не является ли понятие неравновесной энтропии лишним?", чтобы затем ответить, что к энтропии настолько привыкли, что хотелось бы ее сохранить как важную физическую величину. Он также указывает на трудности определения энтропии, если нет ясного определения физического состояния системы. Главная трудность, как было отмечено Брикмонтом, состоит в том, что мы можем ввести столько энтропий, сколько сможем найти наборов макроскопических переменных. Кроме того, из-за процедуры "огрубления" нет резкой границы между микроскопией и макроскопией (между ними - мезоскопия), так что мы можем получить много значений для "энтропии", включая нулевое значение, когда используется полностью микроскопическое описание, т. е. мы имеем чисто механическое описание и термодинамика не существует (см. [117]--[119], а также книгу Джейнса [95]). Джейнс справедливо утверждает, что он не знает, чему равна энтропия кошки; проблема в том, что мы не можем точно определить набор макропеременных, которые правильно определяют термодинамическое состояние кошки.

Приведем, с некоторыми изменениями, несколько совершенно уместных замечаний, сделанных Брикмонтом.

1. Эти энтропии являются не "субъективными", а “объективными" величинами, точно так же, как и соответствующие макроскопические переменные. Названные Джейнсом "антропоморфическими”, согласно Вигнеру, они скорее являются "контекстуальными", т. е. зависят от физической ситуации и уровня ее описания.

2. "Обычная" (или "традиционная") энтропия Клаузиуса соответствует частному выбору макроскопических переменных для свободного моноатомного газа в равновесии (энергии, удельного объема, числа частиц). Дифференциал этой энтропии при изменении энергии определяет обратную абсолютную температуру Кельвина.

3. Второй закон термодинамики в форме "Энтропия возрастает" становится неопределенным: о какой энтропии идет речь? Если речь идет о двух состояниях равновесия, то это энтропия Клаузиуса. В других случаях, как уже отмечалось, утверждение не является хорошо определенным.

4. Какой бы ни был выбран функциональный вид для энтропии, в большинстве случаев ее трудно вычислить или оценить. Необходимо начать с уравнений эволюции для выбранных базисных переменных и решить их при подходящих начальных и граничных условиях. Кроме того, необратимость, характеризуемая некоторой $\mathcal{H}$-теоремой (типа теоремы Больцмана), не связывает напрямую $\mathcal{H}$-функцию с величиной, которую можно принять за энтропию. Она лишь гарантирует, что выбор начальных условий и некоторая специальная немеханическая гипотеза (типа гипотезы молекулярного хаоса - "Stosszahlanzatz") приводят к появлению стрелы времени и релаксации к конечному равновесию.

5. Теорема Лиувилля об инвариантности объема в фазовом пространстве не приводит к затруднениям, это чисто механический результат. На статистическом уровне размер области, занимаемой фазовыми точками, совместимыми с макроскопическим описанием, изменяется, так как эти ограничения меняются со временем и, следовательно, меняется характеристический набор фазовых точек. Эволюция такого набора отличается от набора траекторий заданных точек в области фазового пространства, объем которой действительно сохраняется в силу теоремы Лиувилля. 
6. Кроме того, мы уже обращали внимание на то, что энтропия Гиббса на самом деле не зависит от времени, поскольку в ФНСА сохраняется начальная информация, и назвали ее мелкозернистой энтропией. Однако, как показано на рис. 1, по прошествии некоторого времени микросостояние системы оказывается вне информационного подпространства и больше не описывается набором базисных переменных, что приводит к потере информации. Возникает необходимость вводить в каждый момент времени проецирование на информационное подпространство, что также показано на рис. 1. Конечно, равновесие является частным случаем, когда энтропия Гиббса совпадает с термодинамической энтропией Клаузиуса и в ФНСП всегда остается равной своему значению в начальный момент приготовления.

7. Брикмонт делает заявление, аналогичное заявлению Мейкснера: почему следует так беспокоиться об энтропии неравновесных состояний? Для того чтобы объяснить необратимое поведение макроскопических переменных, необязательно вводить некую функцию энтропии, которая монотонно изменяется со временем. Необратимость не требуется объяснять, однако это может оказаться интересным или полезным. В изложенном ФНСА информационная энтропия применяется к следующим интересным и важным вопросам: она позволяет лучше прояснить смысл и интерпретацию неравновесных термодинамических переменных; позволяет ввести производство информационной энтропии и вывести полезные критерии эволюции и устойчивости; она используется для характеристики диссипативных процессов в среде (упорядочивая их по росту средних отклонений оператора производства информационной энтропии); она помогает анализировать флуктуации в неравновесных состояниях и изучать дополнительность микро- и макроописаний; и т. д.

Поэтому, в принципе и по всей видимости, истинная энтропия четко определена в подходе Клаузиуса только в строго равновесных условиях. В неравновесных ситуациях могут быть введены и оказаться полезными квазиэнтропии (по нашей терминологии), однако требуется ясно указать, как определена квазиэнтропия, и подобрать подходящий термин, который ее характеризует, скажем энтропия в классической (онсагеровской) термодинамике необратимых процессов, энтропия в расширенной термодинамике необратимых процессов, представленная здесь СТНП-энтропия и т. д.

Подводя итог, можно утверждать, что ФНСА представляет собой надежный формализм для построения термодинамики необратимых процессов на статистико-механической основе - подход, названный СТНП. Таким образом, для систем, находящихся в совершенно произвольных диссипативных условиях (без предположения о локальности, линейности и т. д.), имеется теоретическое описание широкого класса термодинамических и транспортных свойств, а также неравновесных функций отклика. Перефразируя слова Цванцига [27], отметим, что, по-видимому, ФНСА обладает замечательной компактностью и имеет безусловно привлекательную структуру, являясь очень эффективным методом изучения нелинейных и нелокальных в пространстве и во времени процессов переноса в сильно неравновесных многочастичных системах.

В заключение упомянем о так называемых теоретико-информационных энтропиях, которые иногда ошибочно рассматриваются как физические энтропии и связаны с так называемой новой неаддитивной термодинамикой [123]. 
Напомним, что в статистической механике распределение вероятностей (статистический оператор), обычно получаемое на основе эвристических аргументов, может быть также выведено из принципа экстремума, как только установлена связь с теорией информации [124]. Вывод состоит в нахождении максимума, при некоторых ограничениях, функционала (супероператора) от распределения вероятностей (статистического оператора). Такого рода величину, впервые введенную в теории связи Шеннона [125]-[127], можно назвать мерой неопределенности информаuии. Ее также называют статистической мерой и энтропией, подразумевая, что это теоретико-информационная энтропия. Стоит подчеркнуть (ввиду некоторого недоразумения, распространенного в последнее время в научной литературе), что различные возможные теоретико-информационные энтропии не следует интерпретировать как термодинамическую энтропию физической системы. Кокс [128] заметил, что смысл такого рода энтропий во всех отношениях отличается от смысла всего того, что имеет близкое название в обычной практике, поэтому для этих величин невозможно дать простое словесное описание, которое в то же время является правильным определением. Джейнс [58] также отметил, что это неудачная терминология и основная "профессиональная болезнь" заключается в "настойчивом отказе различать информационную энтропию, которая является свойством любого распределения вероятностей, и экспериментальную термодинамическую энтропию, которая, вместо этого, является свойством термодинамического состояния... Многие учебники и научные статьи фатально ошибочны из-за нежелания авторов различать эти совершенно разные понятия..." (дальнейшее рассмотрение см. в [129]-[132]).

С чувством печали и скорби мы выражаем глубокое сожаление в связи с кончиной нашего дорогого коллеги проф. др. Ауреа Розы Васконселлос (Áurea Rosas Vasconcellos), подлинного, увлеченного и в высшей степени компетентного преподавателя и исследователя с горячей преданностью теоретической физике конденсированного состояния. Она внесла важный вклад в развитие информационно-статистической термодинамики, описанной в настоящей работе.

\section{Список литературы}

[1] P. W. Anderson, "More is different. Broken symmetry and the nature of the hierarchical structure of science", Science, 4047 (1972), 393-396.

[2] P. W. Anderson, "Is Complexity Physics? Is it Science? What is it?", Phys. Today, 44:7 (1991), 9-10.

[3] R. Luzzi, A. R. Vasconcellos, "Complex behavior in condensed matter: morphological order in dissipative carrier system", Complexity, 2:5 (1997), 42-49.

[4] M. V. Mesquita, A. R. Vasconcellos, R. Luzzi, "Complexity in biological systems", Contemp. Phys., 40:4 (1999), 247-256.

[5] Г. Николис, И. Пригожин, Познание сложного. Введение, Едиториал УРСС, 2013.

[6] R. Luzzi, A. R. Vasconcellos, J. G. Ramos, Statistical Foundations of Irreversible Thermodynamics, Teubner-Texte zur Physik [Teubner Texts in Physics], 35, Teubner, Stuttgart, 2000.

[7] P. T. Landsberg, "Foundations of thermodynamics", Rev. Modern Phys., 28:4 (1956), 363-392.

[8] A. Hobson, "Irreversibility and information in mechanical systems", J. Chem. Phys., 45:4 (1966), 1352-1357. 
[9] B. C. Eu, Kinetic Theory of Irreversible Thermodynamics, Wiley, New York, 1992.

[10] R. V. Velasco, L.S. Garcia-Colin, "The kinetic foundations of non-local nonequilibrium thermodynamics", J. Non-Equilib. Thermodyn., 18:2 (1993), 157.

[11] R. E. Nettleton, "Generalized Grad-type foundations for nonlinear extended thermodynamics", Phys. Rev. A, 42:8 (1990), 4622-4629.

[12] L.S. García-Colín, M. López de Haro, R. F. Rodríguez, J. Casas-Vázquez, D. Jou, "On the foundations of extended irreversible thermodynamics", J. Statist. Phys., 37:3-4 (1984), $465-484$.

[13] Д. Н. Зубарев, Неравновесная статистическая термодинамика, Наука, М., 1971.

[14] D. N. Zubarev, "The method of the non-equilibrium statistical operator and its application. I. The non-equilibrium statistical operator", Fortschr. Physik, 18 (1970), 125-147.

[15] Д. Н. Зубарев, "Современные методы статистической теории неравновесных процессов", Итоги науки и техн. Сер. Соврем. пробл. матем., 15, ВИНИТИ, М., 1980, 131-226.

[16] D. N. Zubarev, "Modern methods of the statistical theory of nonequilibrium processes", J. Soviet Math., 16:6 (1981), 1509-1571.

[17] R. Luzzi, Á. R. Vasconcellos, J. Galväo Ramos, Predictive Statistical Mechanics. A Nonequilibrium Statistical Ensemble Formalism, Fundamental Theories of Physics, 122, Kluwer, Dordrecht, 2002.

[18] Д. Н. Зубарев, В. Г. Морозов, Г. Рёпке, Статистическая механика неравновесных процессов, т. 1, Физматлит, М., 2002.

[19] Д. Н. Зубарев, В. Г. Морозов, Г. Рёпке, Статистическая механика неравновесных процессов, т. 2, Физматлит, М., 2002.

[20] А.И. Ахиезер, С. В. Пелетминский, Методъ статистической физики, Наука, М., 1977.

[21] J. A. McLennan, "The formal statistical theory of transport processes", Advances in Chemical Physics, 5, ed. I. Prigogine, Academic Press, New York, 1963, 261-317.

[22] W. T. Grandy, Principles of Statistical Mechanics, v. 1: Equilibrium Theory, Reidel, Dordrecht, 1987.

[23] W. T. Grandy, Principles of Statistical Mechanics, v. 2: Nonequilibrium Phenomena, Reidel, Dordrecht, 1988.

[24] B. Robertson, "Equations of motion in nonequilibrium statistical mechanics", Phys. Rev., 144:1 (1996), 151-161.

[25] J.P. Dougherty, "Foundations of non-equilibrium statistical mechanics", Philos. Trans. Roy. Soc. London Ser. A, 346:1680 (1994), 259-305.

[26] R. Luzzi, A.R. Vasconcellos, "On the nonequilibrium statistical operator method", Fortschr. Phys., 38:11 (1990), 887-922.

[27] R. Zwanzig, "Where do we go from here?", Perspectives in Statistical Mechanics, ed. H. J. Raveché, North Holland, Amsterdam, 1981, 123-124.

[28] J. G. Kirkwood, "The statistical mechanical theory of transport processes I. General theory", J. Chem. Phys., 14 (1946), 180-201.

[29] M.S. Green, "Markoff random processes and the statistical mechanics of time-dependent phenomena", J. Chem. Phys., 20 (1952), 1281-1295.

[30] H. Mori, I. Oppenheim, J. Ross, "Some topics in quantum statistics. The Wigner function and transport theory", Studies in Statistical Mechanics, v. I, eds. J. de Boer, G. E. Uhlenbeck, North Holland, Amsterdam, 1962, 213-298.

[31] H. Mori, "Transport, collective motion, and Brownian motion", Prog. Theor. Phys., 33:3 (1965), 423-455.

[32] R. Zwanzig, "Statistical mechanics of irreversibility", Lectures in Theoretical Physics, v. 3, eds. W. E. Brittin, B. W. Downs, J. Downs, Wiley-Interscience, New York, 1961, 106-141. 
[33] Н.Н. Боголюбов, Проблемы динамической теории в статистической физике, Гостехиздат, М., Л., 1946.

[34] R. Peierls, "Some simple remarks on the basis of transport theory", Transport Phenomena (Sitges, Barcelona, June 1974), Lecture Notes in Physics, 31, eds. G. Kirczenow, J. Marro, Springer, Berlin, 1974, 1-33.

[35] U. Fano, "Description of states in quantum mechanics by density matrix and operator techniques", Rev. Modern Phys., 29 (1957), 74-93.

[36] B. Robertson, "Introduction to field operators in quantum mechanics", Amer. J. Phys., 41:5 (1973), 678-690.

[37] Р. Фейнман, Статистическая механика, Мир, М., 1975.

[38] P. L. Taylor, A Quantum Approach to the Solid State, Prentice Hall, Englewood Cliffs, NJ, 1970.

[39] J. M. Ziman, Elements of Advanced Quantum Theory, Cambridge Univ. Press, Cambridge, 1969.

[40] J. Klauder, B. Skagerstam, Coherent States, World Sci., Singapore, 1984.

[41] N. Hugenholtz, "Applications of field theoretical methods to many-boson systems", Cargèse Lectures in Theoretical Physics, Lecture III, ed. M. Lévy, Benjamin, New York, 1963, 31 pp.

[42] Н. С. Крылов, Работы по обоснованию статистической физики, Из-во АН СССР, М., 1950.

[43] N. N. Bogoliubov, Lectures in Quantum Mechanics, v. 1, Gordon and Breach, New York, 1967.

[44] N. N. Bogoliubov, Lectures in Quantum Mechanics, v.2, Gordon and Breach, New York, 1970.

[45] В. С. Владимиров, А. А. Логунов, "Памяти Николая Николаевича Боголюбова (21.08.1909 - 13.03.1992)", ТМФ, 92:2 (1992), 179-181.

[46] L. Lauck, Á. R. Vasconcellos, R. Luzzi, "A nonlinear quantum transport theory", Phys. A, 168:2 (1990), 789-819.

[47] J.R. Madureira, Á. R. Vasconcellos, R. Luzzi, L. Lauck, "Markovian kinetic equations in a nonequilibrium statistical ensemble formalism", Phys. Rev. E, 57:3 (1998), 3637-3640.

[48] J. R. Madureira, A. R. Vasconcellos, R. Luzzi, J. Casas-Vazquez, D. Jou, "Evolution of dissipative processes via a statistical thermodynamic approach. I. Generalized Mori-Heisenberg-Langevin equations", J. Chem. Phys., 108:18 (1998), 7568-7579.

[49] J. G. Ramos, A. R. Vasconcellos, R. Luzzi, "A classical approach in predictive statistical mechanics: a generalized Boltzmann formalism", Fortschr. Phys., 43:4 (1995), 265-300.

[50] F. S. Vannucchi, Á.R. Vasconcellos, R. Luzzi, "Thermo-statistical theory of kinetic and relaxation processes", Internat. J. Modern Phys. B, 23:27 (2009), 5283-5305.

[51] B. Robertson, "Equations of motion in nonequilibrium statistical mechanics. II. Energy transport", Phys. Rev., 160:1 (1967), 175-183; Erratum 166:1 (1968), 206.

[52] C. A. B. Silva, Á. R. Vasconcellos, J. G. Ramos, R. Luzzi, "Generalized kinetic equations for far-from-equilibrium many-body systems", J. Statist. Phys., 143:5 (2011), 1020-1034.

[53] H. Spohn, "Kinetic equations from Hamiltonian dynamics: Markovian limits", Rev. Modern Phys., 52:3 (1980), 569-615.

[54] Ю.Л. Климонтович, Статистическая теория открытых систем, ТОО "Янус", М., 1995.

[55] W. M. Elsasser, "On quantum measurements and the role of the uncertainty relations in statistical mechanics", Phys. Rev., 52:9 (1937), 987-999.

[56] E. T. Jaynes, "A backward look to the future", Physics and Probability, eds. W. T. Grandy, P. W. Milonni, Cambridge Univ. Press, Cambridge, 1993, 261-275. 
[57] E. T. Jaynes, "Notes on present status and future prospects", Maximum Entropy and Bayesian Methods (Laramie, Wyoming, 1990), Fundamental Theories of Physics, 43, eds. W. T. Grandy, L. H. Schick, Springer, Netherlands, 1991, 1-13.

[58] E. T. Jaynes, Probability Theory: The Logic of Science, Cambridge Univ. Press, Cambridge, 2002.

[59] E. B. Davies, "Markovian master equations", Commun. Math. Phys., 39:2 (1994), 91-110.

[60] Р. Курант, Д. Гильберт, Методы математической физики, Государственное издательство технико-теоретической литературы, М., Л., 1951.

[61] H. Barnum, C. M. Caves, C. Fuchs, R. Schack, D. J. Driebe, W. G. Hoover, H. Posch, B. L. Holian, R. Peierls, J. L. Lebowitz, "Is Boltzmann entropy time's arrow's archer?", Phys. Today, 47:11 (1994), 11-15.

[62] H.B. Callen, Thermodynamics. An Introduction to the Physical Theories of Equilibrium Thermostatics and Irreversible Thermodynamics, John Wiley and Sons, 1960.

[63] S. R. De Groot, P. Mazur, Non-equilibrium Thermodynamics, North Holland, Amsterdam, 1962.

[64] L. Onsager, "Reciprocal relations in irreversible processes. I", Phys. Rev., 37:4 (1931), 405-426.

[65] L. Onsager, "Reciprocal relations in irreversible processes. II", Phys. Rev., 38:12 (1931), 2265-2279.

[66] P. Glansdorff, I. Prigogine, Thermodynamic Theory of Structure, Stability and Fluctuations, Wiley-Interscience, New York, 1971.

[67] L. Tisza, "Concluding remarks", Thermodynamics: History and Philosophy. Facts, Trends, Debates (Veszprém, Hungary, July 23-28, 1990), eds. K. Martinás, L. Ropolyi, P. Szegedi, World Sci., Singapore, 1991, 515-522.

[68] C. Truesdell, Rational Thermodynamics, Springer, Berlin, 1988.

[69] D. Jou, J. Casas-Vázquez, G. Lebon, Extended Irreversible Thermodynamics, Springer, Berlin, 2010.

[70] D. Jou, J. Casas-Vázquez, G. Lebon, "Extended irreversible thermodynamics", Rep. Prog. Phys., 51:8 (1988), 1105-1179.

[71] G. Lebon, D. Jou, J. Casas-Vázquez, "Questions and answers about a thermodynamic theory of the third type", Contemp. Phys., 33:1 (1992), 41-51.

[72] G. Lebon, D. Jou, "Early history of extended irreversible thermodynamics (1953-1983): an exploration beyond local equilibrium and classical transport theory", Eur. J. Phys. H, 40:2 (2015), 205-240.

[73] D. Jou, J. Casas-Vázquez, G. Lebon, "Extended irreversible thermodynamics revisited", Rep. Progr. Phys., 62:7 (1999), 1035-1142.

[74] I. Gyarmati, "On the wave appproach to thermodynamics and some problems of non-linear theories", J. Non-Equilib. Thermodyn., 2:4, 233-260.

[75] M. Grmela, "Thermodynamics of driven systems", Phys. Rev. E, 48:2 (1993), 919-930.

[76] N. Bernardes, "Thermodynamics and complementarity", Phys. A, 260:1-2 (1998), 186-200.

[77] Á. R. Vasconcellos, R. Luzzi, J. G. Ramos, "Irreversible thermodynamics in a nonequilibrium statistical ensemble formalism", La Rivista del Nuovo Cimento, 24:3 (2001), 1-70.

[78] R. Luzzi, Á. R. Vasconcellos, J. G. Ramos, "The theory of irreversible processes: foundations of a non-equilibrium statistical ensemble formalism", La Rivista del Nuovo Cimento, 29:2 (2006), 1-82.

[79] R. Luzzi, Á. R. Vasconcellos, J. G. Ramos, "Non-equilibrium statistical mechanics of complex systems: an overview", La Rivista del Nuovo Cimento, 30:3 (2007), 95-157.

[80] C. A. B. Silva, J. G. Ramos, Á. R. Vasconcellos, R. Luzzi, Nonlinear higher-order hydrodynamics. Unification of kinetic and hydrodynamic approaches within a nonequilibrium statistical ensemble formalism, arXiv: 1210.7280. 
[81] C. G. Rodrigues, Á.R. Vasconcellos, R. Luzzi, "Mesoscopic hydro-thermodynamics of phonons in semiconductors: heat transfer in III-nitrides", Eur. Phys. J. B, 86:5 (2013), 200, 9 pp.

[82] Á. R. Vasconcellos, A. R. B. de Castro, C. A. B. Silva, R. Luzzi, "Mesoscopic hydro-thermodynamics of phonons", AIP Adv., 3:7 (2013), 072106-072133.

[83] C. A. B. Silva, C. G. Rodrigues, J. G. Ramos, R. Luzzi, "Higher-order generalized hydrodynamics: foundations within a nonequilibrium statistical ensemble formalism", Phys. Rev. E, 91:6 (2015), 063011, 15 pp.

[84] C. G. Rodrigues, A. R. B. Castro, R. Luzzi, "Higher-order generalized hydrodynamics of carriers and phonons in semiconductors in the presence of electric fields: macro to nano", Phys. Stat. Sol. B, 252:12 (2015), 2802-2819.

[85] C. G. Rodrigues, Á. R. Vasconcellos, R. Luzzi, "Thermal conductivity in higher-order generalized hydrodynamics: characterization of nanowires of silicon and gallium nitride", Phys. E, 60 (2014), 50-58.

[86] J.L. del Río, L.S. García-Colín, "Repeated randomness assumption and the projection operator formalism", Phys. Rev. E, 54:1 (1996), 950-953.

[87] C. E. Shannon, W. Weaver, The Mathematical Theory of Communication, Univ. Illinois Press, Urbana, IL, 1949.

[88] L. Brillouin, Science and Information Theory, Academic Press, New York, 1962.

[89] I. Prigogine, Étude Thermodinamique des Phénomenès Irreversibles, Dover, Liège, 1947.

[90] S. A. Hassan, A. R. Vasconcellos, R. Luzzi, "Informational-statistical thermodynamics of a dissipative system in a steady state", Physica A, 235:3-4 (1997), 345-368.

[91] E. T. Jaynes, "The evolution of Carnot's principle", Maximum Entropy and Bayesian Methods in Science and Engineering (Laramie, Wyoming, August 5-8, 1985), eds. G. J. Erickson, C. R. Smith, Kluwer, Dordrecht, 1988, 267-281.

[92] L. Rosenfeld, "On the foundations of statistical thermodynamics", Acta Phys. Polon., 14 (1955), 3-29.

[93] L. Rosenfeld, "Questions on irreversibility and ergodicity", Proceedings of the International School of Physics. "Enrico Fermi", Course XIV, ed. P. Caldirola, Academic Press, New York, 1960, 1-20.

[94] R. Luzzi, J. G. Ramos, Á. R. Vasconcellos, "Rosenfed-Prigogine complementarity of descriptions in the context of informational statistical thermodynamics", Phys. Rev. E, 57:1 (1998), 244-251.

[95] E. T. Jaynes, Papers on Probability, Statistics and Statistical Physics, Synthese Library, 158, Reidel, Dordrecht, 1983.

[96] E. T. Jaynes, "Gibbs vs Boltzmann entropies", Amer. J. Phys., 33:5 (1965), 391-399.

[97] Г. Николис, И. Пригожин, Самоорганизация в неравновесных системах: от диссипативных структур к упорядоченности через флуктуачии, Мир, М., 1979.

[98] G. Nicolis, "Dissipative systems", Rep. Progr. Phys., 49:8 (1986), 873-949.

[99] D. Jou, J. Casas-Vázquez, "Possible experiment to check the reality of a nonequilibrium temperature", Phys. Rev. A, 45:12 (1992), 8371-8373.

[100] R. Luzzi, Á. R. Vasconcellos, J. Casas-Vázquez, D. Jou, "On the selection of the state space in nonequilibrium thermodynamics", Phys. A, 248:1-2 (1998), 111-137.

[101] R. Luzzi, Á. R. Vasconcellos, "Response function theory for far-from-equilibrium systems", J. Statist. Phys., 23:5 (1980), 539-559.

[102] Á. R. Vasconcellos, R. Luzzi, D. Jou, J. Casas-Vázquez, "Thermodynamic variables in the context of a nonequilibrium statistical ensemble approach", J. Chem. Phys., 107:18 (1997), 7383-7396. 
[103] A. C. Algarte, Á. R. Vasconcellos, R. Luzzi, "Kinetics of hot elementary excitations in photoexcited polar semiconductors", Phys. Stat. Sol. B, 173 (1992), 487-514.

[104] A.C. Algarte, A.R. Vasconcellos, R. Luzzi, "Ultrafast kinetics of evolution of optical phonons in a photoinjected highly excited plasma in semiconductors", Phys. Rev. B, 54:16 (1996), 11311-11316.

[105] A. C. Algarte, A. R. Vasconcellos, R. Luzzi, "Ultrafast phenomena in the photoinjected plasma in semiconductors", Braz. J. Phys., 26 (1996), 543-552.

[106] A. C. Algarte, A. R. Vasconcellos, R. Luzzi, "Cooling of hot carriers in highly photoexcited semiconductors", Phys. Rev. B, 38:3 (1988), 2162-2165.

[107] N. Bohr, "On the notions of causality and complementarity", Dialectica, 2:3-4 (1948), $312-319$.

[108] И. Пригожин, От существующего к возникающему. Время и сложность в физических науках, Наука, М., 2002.

[109] H. Atlan, Entre le cristal et la fumèe. Essai sur l'organisation du vivant, Seuil, Paris, 1986.

[110] I. Prigogine, I. Stengers, Order out of Chaos, Bantam, New York, 1984.

[111] R. Landauer, "Information is physical", Phys. Today, 44:5 (1991), 23-31.

[112] S. J. Kline, N. Rosenberg, "An overview of innovation", The Positive Sum Strategy: Harnessing Technology for Economic Growth, eds. R. Landau, N. Rosenberg, National Acad. Sci., Washington, DC, 1986, 275-305.

[113] E. Lutz, S. Ciliberto, "Information: From Maxwell's demon to Landauer's eraser", Phys. Today, 68:9 (2015), 30-37.

[114] L. Szilard, "Über die Entropieverminderung in einem thermodynamischen System bei Eingriffen intelligenter Wesen", Z. Physik, 53:11-12 (1929), 840-856.

[115] C. H. Bennett, "The thermodynamics of computation - a review", Internat. J. Theor. Phys., 21:12 (1982), 905-940.

[116] L. Sklar, Physics and Chance: Philosophical Issues in the Foundations of Statistical Mechanics, Cambridge Univ. Press, Cambridge, 1993.

[117] J. Bricmont, D. Dürr, M. C. Galavotti, G. Ghirardi, F. Petruccione, N. Zanghi, Chance in Physics: Foundations and Perspectives, Lecture Notes in Physics, 574, Springer, Heidelberg, 2001.

[118] J. Bricmont, "Science of chaos or chaos in science?", Physicalia Magazine, 17:3-4 (1995), 159-208.

[119] J. Bricmont, "Science of chaos or chaos in science?", Ann. New York Academy of Sciences, 775 (1996), 131-175.

[120] J. Meixner, "The entropy problem in thermodynamic processes", Rheologica Acta, 12:3 (1973), 465-467.

[121] J. Meixner, "Entropy and entropy production", Foundations of Continuum Thermodynamics, eds. J. J. Delgado, M. N. Nina, J. H. Whitelaw, MacMillan, London, 1974, 129-141.

[122] J. Meixner, H. G. Reik, "Thermodynamik der irreversiblen Prozesse", Principles of Thermodynamics and Statistics. Handbuch der Physik, v. 3, ed. S. Flügge, Springer, Berlin, 1959, $413-523$.

[123] S. Abe, Y. Okamoto (eds.), Nonextensive Statistical Mechanics and its Applications (Okazaki, February 15-18, 1999), Lecture Notes in Physics, 560, Springer, Berlin, 2001.

[124] J. N. Kapur, H. K. Kesavan, Entropy Optimization Principles with Applications, Academic Press, Boston, 1992.

[125] C. E. Shannon, "A mathematical theory of communication I", Bell Syst. Tech. J., 27:3 (1948), 379-423.

[126] C. E. Shannon, "A mathematical theory of communication II", Bell Syst. Tech. J., 27:4 (1948), 623-656. 
[127] N. J. A. Sloane, A. D. Wyner (eds.), Claude Elwood Shannon. Collected Papers, WileyInterscience, New York, 1993.

[128] R. T. Cox, The Algebra of Probable Inference, The Johns Hopkins Univ. Press, Baltimore, 1961.

[129] A. Cho, "A fresh take on disorder, or disorderly science?", Science, 297:5585 (2002), $1268-1269$.

[130] R. Luzzi, Á. R. Vasconcellos, J. G. Ramos, "Trying to make sense of disorder", Science, 298:5596 (2002), 1171-1172.

[131] R. Luzzi, Á. R. Vasconcellos, J. G. Ramos, "Letter to the Editor: 'On fallacies concerning nonextensive thermodynamics and q-entropy"', Europhys. News, 37:2 (2006), 11.

[132] R. Balian, M. Nauenberg, "Letter to the Editor", Europhys. News, 37:2 (2006), 9.

Поступила в редакцию 1.03.2017, после доработки 27.03.2017 